\title{
A New Modified Hybrid Steepest-Descent by Using a Viscosity Approximation Method with a Weakly Contractive Mapping for a System of Equilibrium Problems and Fixed Point Problems with Minimization Problems
}

\author{
Uamporn Witthayarat, ${ }^{1}$ Thanyarat Jitpeera, ${ }^{2}$ and Poom Kumam ${ }^{1}$ \\ ${ }^{1}$ Department of Mathematics, Faculty of Science, King Mongkut's University of Technology Thonburi \\ KMUTT, Bangkok 10140, Thailand \\ ${ }^{2}$ Department of Mathematics and Statistics, Faculty of Science and Agricultural Technology, Rajamangala \\ University of Technology Lanna Chiang Rai, Chiang Rai 57120, Thailand
}

Correspondence should be addressed to Poom Kumam, poom.kum@kmutt.ac.th

Received 8 July 2012; Revised 29 August 2012; Accepted 29 August 2012

Academic Editor: Yongfu Su

Copyright @ 2012 Uamporn Witthayarat et al. This is an open access article distributed under the Creative Commons Attribution License, which permits unrestricted use, distribution, and reproduction in any medium, provided the original work is properly cited.

The purpose of this paper is to consider a modified hybrid steepest-descent method by using a viscosity approximation method with a weakly contractive mapping for finding the common element of the set of a common fixed point for an infinite family of nonexpansive mappings and the set of solutions of a system of an equilibrium problem. The sequence is generated from an arbitrary initial point which converges in norm to the unique solution of the variational inequality under some suitable conditions in a real Hilbert space. The results presented in this paper generalize and improve the results of Moudafi (2000), Marino and Xu (2006), Tian (2010), Saeidi (2010), and some others. Finally, we give an application to minimization problems and a numerical example which support our main theorem in the last part.

\section{Introduction}

The convex feasibility problem (CFP) is the problem for finding points in the intersection of a finite family of closed convex subsets $C_{i}, i=1,2, \ldots, N$ in the framework of Hilbert spaces, that is, to find a point $\widehat{x}$ such that

$$
\widehat{x} \in \bigcap_{i=1}^{N} C_{i} .
$$


This problem plays an extremely important role in various fields, especially in applied mathematics and physical sciences; moreover, it has a great impact role on the real-world applications (see [1, 2]). The well-known applications are the theory of optimization $[3,4]$, image reconstruction by the projection method [5], signal processing problems [6], and model for the problem in sensor networks [7], as some powerful examples.

We focus on the important subclass of convex feasibility problems, in which finitely many sets are given. Each set can be specified in various forms, such as the fixed point set of a nonexpansive mapping, the set of solutions of the variational inequality, and the set of solutions to an equilibrium problem. In a framework of Hilbert spaces, there are some applications of convex feasibility problems in various disciplines such as image restoration, computer tomograph, and radiation therapy treatment planning [8].

Throughout this paper, we assume that $H$ is a real Hilbert space with inner product $\langle\cdot, \cdot\rangle$ and norm $\|\cdot\|$, and let $C$ be a nonempty closed convex subset of $H$. Let $\Im=\left\{F_{j}\right\}_{j \in \Gamma}$ be bifunctions from $C \times C$ to $\mathbb{R}$, where $\mathbb{R}$ is the set of real numbers, and $\Gamma$ is an arbitrary index set. The system of equilibrium problems is to find $x \in C$ such that

$$
F_{j}(x, y) \geq 0, \quad \forall y \in C, j \in \Gamma \text {. }
$$

If $\Gamma$ is a singleton, then problem (1.2), reduced to the equilibrium problems, is to find $x \in C$ such that

$$
F(x, y) \geq 0, \quad \forall y \in C
$$

The set of solution of (1.3) is denoted by $\operatorname{EP}(F)$. The above formulation (1.3) was shown in [7] to cover monotone inclusion problems, saddle point problems, variational inequality problems, minimization problems, optimization problems, vector equilibrium problems, and Nash equilibria in noncooperative games. In other words, the $\mathrm{EP}(F)$ is a unifying model for several problems arising in physics, engineering, science, optimization, economics, and so forth; Combettes and Hirstoaga [9] introduced an iterative scheme for finding a common element in the solution set of problem (1.3) in a Hilbert space.

The equilibrium problems include fixed point problems, optimization problems, variational inequalities problems, Nash equilibrium problems, noncooperative games, and economics and the equilibrium problems; as special cases see, for example, [7, 10-14]. Some methods have been proposed to solve the equilibrium problem; see, for instance, [15-22].

Let $B: C \rightarrow H$ be a mapping. The variational inequality problem, denoted by $\operatorname{VI}(C, B)$, is to find $x \in C$ such that

$$
\langle B x, y-x\rangle \geq 0, \quad \forall y \in C
$$

Existence and uniqueness of solutions are the most important problems of $\operatorname{VI}(C, B)$. The variational inequality problem has been extensively studied in the literature, see, for example, $[23,24]$ and the references therein. It is known that if $B$ is a strong monotone and Lipschitzian mapping on $C$, then $\operatorname{VI}(C, B)$ has a unique solution. Variational inequalities are among the most interesting and important mathematical problems and have been studied intensively in the past years since they have wide applications in the optimization and control, economics and transportation equilibrium, and engineering science. For these reasons, many 
existence results and iterative algorithms for various variational inclusions have been studied extensively by many authors. For details, see [2, 7, 23-25] and references therein.

On the other hand, iterative methods for nonexpansive mappings have recently been applied to solve convex minimization problems. Convex minimization problems have a great impact and influence in the development of almost all branches of pure and applied sciences.

A mapping $T: C \rightarrow C$ is called nonexpansive if $\|T x-T y\| \leq\|x-y\|$, for all $x, y \in C$. We use $F(T)$ to denote the set of fixed points of $T$, that is, $F(T)=\{x \in C: T x=x\}$. Recall that a self-mapping $f: C \rightarrow C$ is a contractive mapping on $C$ if there exists a constant $\alpha \in[0,1)$ such that $\|f(x)-f(y)\| \leq \alpha\|x-y\|$, for all $x, y \in C$. A mapping $B: H \rightarrow H$ is said to be a $k$-Lipschitzian if there exists a constant $k>0$ such that $\|B x-B y\| \leq k\|x-y\|$, for all $x, y \in C$. The concept of quasi-nonexpansive was introduced by Diaz and Metcalf [26]. The mapping $T$ is said to be quasi-nonexpansive if $\|T x-p\| \leq\|x-p\|$, for all $x \in C$ and $p \in F(T)$.

In 2000, Moudafi [27] introduced the viscosity approximation method for a nonexpansive mapping $T: C \rightarrow C$. Let $f$ be a contraction on $H$, starting with an arbitrary initial $x_{0} \in H$, defining a sequence $\left\{x_{n}\right\}$ recursively by

$$
x_{n+1}=\alpha_{n} f\left(x_{n}\right)+\left(1-\alpha_{n}\right) T x_{n}, \quad \forall n \geq 0,
$$

where $\alpha_{n}$ is a sequence in $(0,1)$. Xu [28] proved that under certain appropriate conditions on $\left\{\alpha_{n}\right\}$, the sequence $\left\{x_{n}\right\}$ generated by (1.5) converges strongly to the unique solution $x^{*} \in C$ of the variational inequality

$$
\left\langle(I-f) x^{*}, x-x^{*}\right\rangle \geq 0, \quad \forall x \in F(T) .
$$

In 2006, Marino and Xu [29] introduced the following iterative scheme:

$$
x_{n+1}=\alpha_{n} \gamma f\left(x_{n}\right)+\left(I-\alpha_{n} A\right) T x_{n}, \quad \forall n \geq 0 .
$$

It was proved that if the sequence $\left\{\alpha_{n}\right\}$ of parameters satisfies appropriate conditions, then the sequence $\left\{x_{n}\right\}$ generated by (1.7) converges strongly to the unique solution of the variational inequality

$$
\left\langle(A-\gamma f) x^{*}, x-x^{*}\right\rangle \geq 0, \quad \forall x \in F(T),
$$

which is the optimality condition for the minimization problem

$$
\min _{x \in F(T)} \frac{1}{2}\langle A x, x\rangle-h(x)
$$

where $h$ is a potential function for $\gamma f$ (i.e., $h^{\prime}(x)=\gamma f(x)$, for $x \in H$ ). Assume $A$ is strongly positive bounded linear operator. It can be referred that there is a constant $\bar{\gamma}>0$ which satisfies the following property:

$$
\langle A x, x\rangle \geq \bar{\gamma}\|x\|^{2}, \quad \forall x \in H .
$$


In 2007, Suzuki [30] extended Moudafi's viscosity approximations with MeirKeeler contractions and presented very simple proofs of Xu's theorems by concidering Moudafi's approximations.

On the other hand, Yamada [31] introduced the following hybrid iterative scheme for finding the variational inequality:

$$
x_{n+1}=T x_{n}-\mu \lambda_{n} B\left(T x_{n}\right), \quad \forall n \geq 0,
$$

where $B$ is $k$-Lipschitzian and $\eta$-strongly monotone operator with $k>0, \eta>0,0<\mu<2 \eta / k^{2}$, then he proved that if $\left\{\lambda_{n}\right\}$ satisfies some appropriate conditions, then $\left\{x_{n}\right\}$ generated by (1.11) converges strongly to the unique solution of variational inequality

$$
\left\langle B x^{*}, x-x^{*}\right\rangle \geq 0, \quad x \in F(T) .
$$

In 2010, Tian [32] combined (1.7) and (1.11) and considered the following general iterative method:

$$
x_{n+1}=\alpha_{n} \gamma f\left(x_{n}\right)+\left(I-\mu \alpha_{n} B\right) T x_{n}, \quad \forall n \geq 0 .
$$

If the sequence $\left\{\alpha_{n}\right\}$ of parameters satisfies appropriate conditions, then the sequence $\left\{x_{n}\right\}$ generated by (1.13) converges strongly to the unique solution $x^{*} \in C$ of the variational inequality

$$
\left\langle(\gamma f-\mu B) x^{*}, x-x^{*}\right\rangle \leq 0, \quad \forall x \in F(T)
$$

Later, Saeidi [33] introduced the following modified hybrid steepest-descent iterative algorithm for finding a common element of the set of solutions of a system of equilibrium problems for a family $\mathcal{F}=\left\{F_{j}: C \times C \rightarrow \mathbb{R}, j=1,2, \ldots, M\right\}$ and the set of common fixed points for a family of infinitely nonexpansive mappings $\mathcal{S}=\left\{S_{i}: C \rightarrow C\right\}$, with respect to $W$-mappings (see (2.14)). The proposed scheme was defined by

$$
\begin{gathered}
y_{n}=W_{n} J_{r_{M}, n}^{F_{M}} \cdots J_{r_{2}, n}^{F_{2}} J_{r_{1}, n}^{F_{1}} x_{n}, \\
x_{n+1}=\beta x_{n}+(1-\beta)\left(I-\lambda_{n} B\right) y_{n}, \quad \forall n \in \mathbb{N},
\end{gathered}
$$

where $B$ is a relaxed $(\gamma, r)$-cocoercive, $k$-Lipschitzian mapping such that $r>\gamma k^{2}$. Then, under weaker hypotheses on coefficients, he proved the strong convergence of the proposed iterative algorithm to the unique solution of the variational inequality. Zhang et al. [34] introduced a modified iterative algorithm by using a viscosity approximation method with a weakly contractive mapping with respect to $W$-mappings (see (2.14)). They defined

$$
x_{n+1}=\alpha_{n} \Phi x_{n}+\left(1-\alpha_{n}\right) W_{n} J_{r_{M}, n}^{F_{M}} \ldots J_{r_{2}, n}^{F_{2}} J_{r_{1}, n}^{F_{1}} x_{n}, \quad \forall n \in \mathbb{N},
$$

where $\Phi$ is a $\pi$-weakly contractive self-mapping on $C$, and $\left\{\alpha_{n}\right\}$ is a sequence in $(0,1)$. They proved that under certain appropriate conditions imposed on $\left\{\alpha_{n}\right\}$, the proposed iterative 
algorithm converges strongly to the common element of the set of common fixed points of an infinite family of nonexpansive mappings and the set of a finite family of equilibrium problems.

In this paper, motivated and inspired by the previously mentioned above results, we consider a modified hybrid steepest-descent method by using a viscosity approximation method with a weakly contractive mapping for finding the common element of the set of common fixed points for an infinite family of nonexpansive mappings with weakly contractive mappings and the set of solutions of a system of equilibrium problems. The sequence generated from an arbitrary initial point $x_{0} \in H$ which will converge in norm to the unique solution of the variational inequality under some suitable conditions in a real Hilbert space. Furthermore, we give an application to minimization problems and a numerical example which support our main theorem in the last part.

\section{Preliminaries}

Let $H$ be a real Hilbert space and $C$ be a nonempty closed convex subset of $H$. We denote weak convergence and strong convergence by notations $\rightarrow$ and $\rightarrow$, respectively. Recall that when the metric (nearest point) projection $P_{C}$ from $H$ onto $C$ assigns to each $x \in H$, the unique point in $P_{C} x \in C$ satisfies the property

$$
\left\|x-P_{C} x\right\|=\min _{y \in C}\|x-y\|
$$

The following characterizes the projection $P_{C}$.

An important problem is how to find a solution of $\mathrm{VI}(C, B)$. It is known that

$$
u \in \mathrm{VI}(C, B) \Longleftrightarrow u=P_{C}(u-\lambda B u)
$$

where $\lambda>0$ is an arbitrarily fixed constant, and $P_{C}$ is the projection of $H$ onto $C$.

We recall some lemmas which will be needed in the rest of this paper.

Lemma 2.1. For a given $z \in H, u \in C$,

$$
u=P_{C} z \Longleftrightarrow\langle u-z, v-u\rangle \geq 0, \quad \forall v \in C
$$

It is well known that $P_{C}$ is a firmly nonexpansive mapping of $H$ onto $C$ and satisfies

$$
\left\|P_{C} x-P_{C} y\right\|^{2} \leq\left\langle P_{C} x-P_{C} y, x-y\right\rangle, \quad \forall x, y \in H
$$

Moreover, $P_{C} x$ is characterized by the following properties: $P_{C} x \in C$ and for all $x \in H, y \in C$,

$$
\left\langle x-P_{C} x, y-P_{C} x\right\rangle \leq 0 .
$$


Definition 2.2. A mapping $\Phi: C \rightarrow H$ with domain $D(\Phi)$ and range $R(\Phi)$ in $H$, Alber and Guerre-Delabriere [35] defined a $\pi$-weakly contractive mapping by the following:

$$
\|\Phi x-\Phi y\| \leq\|x-y\|-\pi(\|x-y\|), \quad \forall x, y \in D(\Phi)
$$

for some $\pi:[0,+\infty) \rightarrow[0,+\infty)$ which is a continuous and strictly increasing function such that $\pi$ is positive on $(0,+\infty)$ and $\pi(0)=0$. If $\pi(t) \equiv(1-k) t$, then $\Phi$ is said to be contractive mapping with the contractive coefficient $k$. If $\pi(t)=0$, then $\Phi$ is said to be nonexpansive. If $\pi(t)=0$ and $y=\Phi y$, then $\Phi$ with a fixed point $y$ is said to be qusi-nonexpansive.

Definition 2.3. A mapping $B: C \rightarrow H$ is said to be an $\eta$-strongly monotone if there exists a constant $\eta>0$ with the following property:

$$
\langle B x-B y, x-y\rangle \geq \eta\|x-y\|^{2}, \quad \forall x, y \in C
$$

Definition 2.4. A mapping $B: C \rightarrow H$ is said to be relaxed $(\gamma, r)$-cocoercive if there exist two constants $r>0$ and $r>0$ which satisfies the following property:

$$
\langle B x-B y, x-y\rangle \geq-r\|B x-B y\|^{2}+r\|x-y\|^{2}, \quad \forall x, y \in C .
$$

Lemma 2.5 (see [28]). Assume that $\left\{a_{n}\right\}$ is a sequence of nonnegative real numbers such that

$$
a_{n+1} \leq\left(1-l_{n}\right) a_{n}+\sigma_{n,}, n \geq 0,
$$

where $\left\{l_{n}\right\}$ is a sequence in $(0,1)$, and $\left\{\sigma_{n}\right\}$ is a sequence in $\mathbb{R}$ such that

(1) $\sum_{n=1}^{\infty} l_{n}=\infty$,

(2) $\lim \sup _{n \rightarrow \infty} \sigma_{n} / l_{n} \leq 0$ or $\sum_{n=1}^{\infty}\left|\sigma_{n}\right|<\infty$.

Then $\lim _{n \rightarrow \infty} a_{n}=0$.

Lemma 2.6 (see [36]). Let $C$ be a closed convex subset of a real Hilbert space $H$ and let $T: C \rightarrow C$ be a nonexpansive mapping. Then $I-T$ is demiclosed at zero, that is, $x_{n} \rightarrow x, x_{n}-T x_{n} \rightarrow 0$ implies $x=T x$.

Lemma 2.7 (see [37]). Let $C$ be a closed convex subset of $H$. Let $\left\{x_{n}\right\}$ be a bounded sequence in $H$. Assume that

(1) the weak $\omega$-limit set $\omega_{w}\left(x_{n}\right) \subset C$,

(2) for each $z \in C, \lim _{n \rightarrow \infty}\left\|x_{n}-z\right\|$ exists.

Then $\left\{x_{n}\right\}$ is weakly convergent to a point in $C$. 
Lemma 2.8 (see [38]). Each Hilbert space $H$ satisfies Opial's condition, that is, for any sequence $\left\{x_{n}\right\} \subset H$ with $x_{n} \rightarrow x$, the inequality,

$$
\liminf _{n \rightarrow \infty}\left\|x_{n}-x\right\|<\liminf _{n \rightarrow \infty}\left\|x_{n}-y\right\|
$$

holds for each $y \in H$ with $y \neq x$.

Lemma 2.9 (see [39]). Each Hilbert space $H$ satisfies the Kadec-Klee property, that is, for any sequence $\left\{x_{n}\right\}$ with $x_{n} \rightarrow x$ and together with $\left\|x_{n}\right\| \rightarrow\|x\|$ implies $\left\|x_{n}-x\right\| \rightarrow 0$.

For solving the equilibrium problem, let us give the following assumptions for a bifunction $F$ of $C \times C$ into $\mathbb{R}$ which were imposed in $[9,40]$ :

(A1) $F(x, x)=0$ for all $x \in C$;

(A2) $F$ is monotone, that is, $F(x, y)+F(y, x) \leq 0$ for all $x, y \in C$;

(A3) for each $x, y, z \in C, \lim _{t \downarrow 0} F(t z+(1-t) x, y) \leq F(x, y)$;

(A4) for each $x \in C, y \mapsto F(x, y)$ is convex and lower semicontinuous.

Lemma 2.10 (see $[9,40])$. Let $C$ be a nonempty closed convex subset of $H$, and let $F$ be a bifunction of $C \times C$ into $\mathbb{R}$ satisfying (A1)-(A4). If $r>0$ and $x \in H$, then there exists $z \in C$ such that

$$
F(z, y)+\frac{1}{r}\langle y-z, z-x\rangle \geq 0
$$

Lemma 2.11 (see [9]). Let $C$ be a nonempty closed convex subset of $H$, and let $F$ be a bifunction of $C \times C$ into $\mathbb{R}$ satisfying (A1)-(A4). For $r>0$ and $x \in H$, define a mapping $J_{r}^{F}: H \rightarrow C$ as follows:

$$
J_{r}^{F}(x)=\left\{z \in C: F(z, y)+\frac{1}{r}\langle y-z, z-x\rangle \geq 0, \forall y \in C\right\}
$$

for all $x \in H$. Then, the following conclusions hold that

(1) $J_{r}^{F}$ is single-valued;

(2) $J_{r}^{F}$ is firmly nonexpansive, that is, for any $x, y \in H$,

$$
\left\|J_{r}^{F}(x)-J_{r}^{F}(y)\right\|^{2} \leq\left\langle J_{r}^{F}(x)-J_{r}^{F}(y), x-y\right\rangle
$$

(3) $F\left(J_{r}^{F}\right)=\mathrm{EP}(F)$;

(4) $\mathrm{EP}(F)$ is closed and convex. 
A family of nonexpansive mappings has been considered by many authors (see [4152] and references therein). Recently, Shang et al. [47] improved the results of Kim and Xu [53] from a single mapping to a finite family of mappings in the framework of Hilbert spaces. Now, we consider the mapping $W_{n}$ defined, as in Shimoji and Takahashi [48], by

$$
\begin{aligned}
& U_{n, n+1}:=I, \\
& U_{n, n}:= \gamma_{n} S_{n} U_{n, n+1}+\left(1-\gamma_{n}\right) I, \\
& U_{n, n-1}:=\gamma_{n-1} S_{n-1} U_{n, n}+\left(1-\gamma_{n-1}\right) I, \\
& \vdots \\
& U_{n, k}:= \gamma_{k} S_{k} U_{n, k+1}+\left(1-\gamma_{k}\right) I, \\
& U_{n, k-1}:=\gamma_{k-1} S_{k-1} U_{n, k}+\left(1-\gamma_{k-1}\right) I, \\
& \quad \vdots \\
& U_{n, 2}:=\gamma_{2} S_{2} U_{n, 3}+\left(1-\gamma_{2}\right) I, \\
& W_{n}:=U_{n, 1}=\gamma_{1} S_{1} U_{n, 2}+\left(1-\gamma_{1}\right) I,
\end{aligned}
$$

where $\gamma_{n}, \gamma_{n-1}, \ldots, \gamma_{1}$ are real numbers such that $0 \leq \gamma_{n} \leq 1$ and $S_{1}, S_{2}, \ldots$ are an infinite family of mappings of $H$ into itself. Nonexpansivity of each $S_{i}$ ensures the nonexpansivity of $W_{n}$.

Lemma 2.12 (see [48]). Let $H$ be a real Hilbert space $H$. Let $S_{1}, S_{2}, \ldots$ be nonexpansive mappings from $H$ into itself such that $\bigcap_{n=1}^{\infty} F\left(S_{n}\right) \neq \emptyset$ and $\left\{\gamma_{1}, \gamma_{2}, \ldots\right\}$ are real numbers such that $0<\gamma_{n} \leq b<1$, for all $n \geq 1$. Then, for every $x \in H$ and $k \in N$, the limit $\lim _{n \rightarrow \infty} U_{n, k} x$ exists.

Using Lemma 2.12, one can define the mapping $W$ from $H$ into itself as follows:

$$
\lim _{n \rightarrow \infty} W_{n} x=\lim _{n \rightarrow \infty} U_{n, 1} x, \quad \forall x \in H .
$$

Such $W$ is called the $W$-mapping generated by $S_{1}, S_{2}, \ldots$ and $\gamma_{1}, \gamma_{2}, \ldots$

Lemma 2.13 (see [48]). Let $H$ be a real Hilbert space $H$. Let $S_{1}, S_{2}, \ldots$ be nonexpansive mappings from $H$ into itself such that $\bigcap_{n=1}^{\infty} F\left(S_{n}\right) \neq \emptyset$ and $\left\{\gamma_{1}, \gamma_{2}, \ldots\right\}$ are real numbers such that $0<\gamma_{n} \leq b<1$, for all $n \geq 1$. Then, $F(W)=\bigcap_{n=1}^{\infty} F\left(S_{n}\right)$.

\section{Main Results}

In this section, we will introduce an iterative scheme by using a modified hybrid steepestdescent method for finding the common element of the set of common fixed points for an 
infinite family of nonexpansive mappings with weakly contractive mappings and the set of solutions of a system of equilibrium problems in a real Hilbert space.

Theorem 3.1. Let $C$ be a nonempty closed convex subset of a real Hilbert space $H$, such that $C \pm C \subset$ C. Let $\mathcal{S}=\left\{S_{i}: C \rightarrow C\right\}$ be a family of infinitely nonexpansive mappings, and let $\mathcal{F}=\left\{F_{j}\right.$ : $j=1,2,3, \ldots, M\}$ be a finite family of bifunctions $C \times C$ to $\mathbb{R}$ satisfying (A1)-(A4). Assume that $\Theta:=\left(\bigcap_{i=1}^{\infty} F\left(S_{i}\right)\right) \cap\left(\bigcap_{j=1}^{M} \mathrm{EP}\left(F_{j}\right)\right) \neq \emptyset$. Let $B$ be a $k$-Lipschitzian and $\eta$-strongly monotone mapping on $C$ with $k>0, \eta>0$. Let $\Phi$ be a $\pi$-weakly contractive self-mapping on $C$ with $\alpha \in[0,1)$. Denote the collection of all weakly contractive $\Phi$ on $C$ by $C$. Let $0<\mu<2 \eta / k^{2}$ and $0<\gamma<\mu\left(\eta-\mu k^{2} / 2\right)=\tau$. Let the mapping $W_{n}$ be defined by (2.14) and $\left\{r_{j, n}\right\}_{j=1}^{M}$ be a sequence in $(0, \infty)$. If $\left\{x_{n}\right\}$ is the sequence generated by $x_{1} \in C$ and

$$
\begin{gathered}
w_{n}=W_{n} J_{r_{M}, n}^{F_{M}} \cdots J_{r_{2}, n}^{F_{2}} J_{r_{1}, n}^{F_{1}} x_{n}, \\
x_{n+1}=\alpha_{n} \gamma \Phi\left(x_{n}\right)+\left(I-\alpha_{n} \mu B\right) w_{n}, \quad \forall n \in \mathbb{N},
\end{gathered}
$$

where $\left\{\alpha_{n}\right\}$ is a sequence in $(0,1)$ and satisfies the following conditions:

(C1) $\lim _{n \rightarrow \infty} \alpha_{n}=0$ and $\sum_{n=1}^{\infty} \alpha_{n}=\infty$;

(C2) $\lim _{n \rightarrow \infty}\left|\alpha_{n+1}-\alpha_{n}\right|=0$;

(C3) $\lim _{n \rightarrow \infty}\left|\gamma_{n+1}-\gamma_{n}\right|=0$;

(C4) $\liminf _{n \rightarrow \infty} r_{j, n}>0$, for all $j \in\{1,2, \ldots, M\}$.

Then, the sequence $\left\{x_{n}\right\}$ converges strongly to $x^{*}=P_{\Theta}(I-\mu B+\gamma \Phi) x^{*}$ which is the unique solution of the variational inequality

$$
\left\langle(\mu B-\gamma \Phi) x^{*}, x^{*}-x\right\rangle \leq 0, \quad \forall x \in \Theta
$$

which is the optimality condition for the minimization problem

$$
\min _{x \in F(T)} \frac{1}{2}\langle B x, x\rangle-h(x)
$$

where $h$ is a potential function for $\gamma \Phi$ (i.e., $h^{\prime}(x)=\gamma \Phi(x)$, for $x \in H$ ).

Proof. We will divide the proof of Theorem 3.1 into several steps.

Step 1. We will show that $\left\{x_{n}\right\}$ is bounded. Let $p \in \Theta$. By taking $\Im_{n}^{k}=J_{r_{k, n}}^{F_{k}} J_{r_{k-1, n}}^{F_{k-1}} J_{r_{k-2, n}}^{F_{k-2}} \cdots J_{r_{2, n}}^{F_{2}} J_{r_{1, n}}^{F_{1}}$ for $k \in\{1,2,3, \ldots, M\}$ and $\Im_{n}^{0}=I$, for all $n \in \mathbb{N}$. Since $J_{r_{k, n}}^{F_{k}}$ is nonexpansive for each $k=$ $1,2,3, \ldots, M$, then, we have

$$
\left\|\Im_{n}^{M} x_{n}-p\right\|=\left\|\Im_{n}^{M} x_{n}-\Im_{n}^{M} p\right\| \leq\left\|x_{n}-p\right\|
$$


From Lemmas 2.11 and 2.12, it follows that

$$
\begin{aligned}
\left\|x_{n+1}-p\right\|= & \left\|\alpha_{n} \gamma \Phi\left(x_{n}\right)+\left(I-\alpha_{n} \mu B\right) w_{n}-p\right\| \\
= & \left\|\alpha_{n} \gamma \Phi\left(x_{n}\right)+\left(I-\alpha_{n} \mu B\right) W_{n} \Im_{n}^{M} x_{n}-p\right\| \\
= & \left\|\alpha_{n}\left(\gamma \Phi\left(x_{n}\right)-\mu B W_{n} \Im_{n}^{M}(p)\right)+\left(I-\alpha_{n} \mu B\right) W_{n} \Im_{n}^{M} x_{n}-\left(I-\alpha_{n} \mu B\right) W_{n} \Im_{n}^{M} p\right\| \\
\leq & \alpha_{n}\left\|\gamma \Phi\left(x_{n}\right)-\gamma \Phi(p)\right\|+\alpha_{n}\left\|\gamma \Phi(p)-\mu B W_{n} \Im_{n}^{M}(p)\right\| \\
& +\left\|\left(I-\alpha_{n} \mu B\right) W_{n} \Im_{n}^{M} x_{n}-\left(I-\alpha_{n} \mu B\right) W_{n} \Im_{n}^{M} p\right\| \\
\leq & \alpha_{n} \gamma\left\{\left\|x_{n}-p\right\|-\pi\left(\left\|x_{n}-p\right\|\right)\right\}+\alpha_{n}\|\gamma \Phi(p)-\mu B(p)\|+\left(1-\alpha_{n} \tau\right)\left\|x_{n}-p\right\| \\
\leq & \left(1-\alpha_{n}(\tau-\gamma)\right)\left\|x_{n}-p\right\|+\alpha_{n}(\tau-\gamma) \frac{\|\gamma \Phi(p)-\mu B(p)\|}{\tau-\gamma} \\
\leq & \max \left\{\left\|x_{n}-p\right\|, \frac{\|\gamma \Phi(p)-\mu B(p)\|}{\tau-\gamma}\right\} .
\end{aligned}
$$

By mathematical induction, it becomes

$$
\left\|x_{n}-p\right\| \leq \max \left\{\left\|x_{1}-p\right\|, \frac{\|\gamma \Phi(p)+\mu B(p)\|}{\tau-\gamma}\right\}, \quad \forall n \geq 1,
$$

and we obtain that $\left\{x_{n}\right\}$ is bounded. So are $\left\{W_{n} \Im_{n}^{M}\left(x_{n}\right)\right\}$ and $\left\{\Phi\left(x_{n}\right)\right\}$.

Step 2. We claim that

$$
\lim _{n \rightarrow \infty}\left\|\Im_{n}^{k} x_{n}-\Im_{n+1}^{k} x_{n}\right\|=0,
$$

for every $k \in\{1,2,3, \ldots, M\}$. From Step 2 of the proof in [54, Theorem 3.1], we have for $k \in\{1,2,3, \ldots, M\}$,

$$
\lim _{n \rightarrow \infty}\left\|J_{r_{k, n+1}}^{F_{k}} x_{n}-J_{r_{k, n}}^{F_{k}} x_{n}\right\|=0 .
$$


Note that for every $k \in\{1,2,3, \ldots, M\}$, we obtain

$$
\Im_{n}^{k}=J_{r_{k, n}}^{F_{k}} J_{r_{k-1, n}}^{F_{k-1}} J_{r_{k-2, n}}^{F_{k-2}} \cdots J_{r_{2, n}}^{F_{2}} J_{r_{1, n}}^{F_{1}}=J_{r_{k, n}}^{F_{k}} \Im_{n}^{k-1}
$$

So, we have

$$
\begin{aligned}
\left\|\Im_{n}^{k} x_{n}-\Im_{n+1}^{k} x_{n}\right\|= & \left\|J_{r_{k, n}}^{F_{k}} \Im_{n}^{k-1} x_{n}-J_{r_{k, n+1}}^{F_{k}} \Im_{n+1}^{k-1} x_{n}\right\| \\
\leq & \left\|J_{r_{k, n}}^{F_{k}} \Im_{n}^{k-1} x_{n}-J_{r_{k, n+1}}^{F_{k}} \Im_{n}^{k-1} x_{n}\right\|+\left\|J_{r_{k, n+1}}^{F_{k}} \Im_{n}^{k-1} x_{n}-J_{r_{k, n+1}}^{F_{k}} \Im_{n+1}^{k-1} x_{n}\right\| \\
\leq & \left\|J_{r_{k, n}}^{F_{k}} \Im_{n}^{k-1} x_{n}-J_{r_{k, n+1}}^{F_{k}} \Im_{n}^{k-1} x_{n}\right\|+\left\|\Im_{n}^{k-1} x_{n}-\Im_{n+1}^{k-1} x_{n}\right\| \\
\leq & \left\|J_{r_{k, n}}^{F_{k}} \Im_{n}^{k-1} x_{n}-J_{r_{k, n+1}}^{F_{k}} \Im_{n}^{k-1} x_{n}\right\|+\left\|J_{r_{k-1, n}}^{F_{k-1}} \Im_{n}^{k-2} x_{n}-J_{r_{k-1, n+1}}^{F_{k-1}} \Im_{n}^{k-2} x_{n}\right\| \\
& +\left\|\Im_{n}^{k-2} x_{n}-\Im_{n+1}^{k-2} x_{n}\right\| \\
\leq & \left\|J_{r_{k, n}}^{F_{k}} \Im_{n}^{k-1} x_{n}-J_{r_{k, n+1}}^{F_{k}} \Im_{n}^{k-1} x_{n}\right\|+\left\|J_{r_{k-1, n}}^{F_{k-1}} \Im_{n}^{k-2} x_{n}-J_{r_{k-1, n+1}}^{F_{k-1}} \Im_{n}^{k-2} x_{n}\right\| \\
& +\cdots+\left\|J_{r_{2, n}}^{F_{2}} \Im_{n}^{1} x_{n}-J_{r_{2, n+1}}^{F_{2}} \Im_{n}^{1} x_{n}\right\|+\left\|J_{r_{1, n}}^{F_{1}} x_{n}-J_{r_{1, n+1}}^{F_{1}} x_{n}\right\| .
\end{aligned}
$$

Now, apply (3.8) to (3.10), we conclude (3.7).

Step 3. We may assume that $B_{n}=\left(I-\alpha_{n} \mu B\right)$. Let $\left\{w_{n}\right\}$ be a bounded sequence in $C$. Then, we show that $\lim _{n \rightarrow \infty}\left\|B_{n+1} w_{n}-B_{n} w_{n}\right\|=0$. Indeed, since $\left\{w_{n}\right\}$ is bounded and $B$ is a Lipschitzian mapping, now, from condition (C2), we have

$$
\begin{aligned}
\left\|B_{n+1} w_{n}-B_{n} w_{n}\right\| & =\left\|\left(I-\alpha_{n+1} \mu B\right) w_{n}-\left(I-\alpha_{n} \mu B\right) w_{n}\right\| \\
& =\left\|\alpha_{n+1} \mu B w_{n}-\alpha_{n} \mu B w_{n}\right\| \\
& =\mu\left|\alpha_{n+1}-\alpha_{n}\right|\left\|B w_{n}\right\| \\
& \leq M_{1}\left|\alpha_{n+1}-\alpha_{n}\right|
\end{aligned}
$$

where $M_{1}$ is an approximate constant such that $M_{1} \geq \max \left\{\sup _{n \geq 1}\left\|B w_{n}\right\|\right\}$. Hence $\| B_{n+1} w_{n}-$ $B_{n} w_{n} \| \rightarrow 0$ as $n \rightarrow \infty$. 
Step 4 . We show that $\lim _{n \rightarrow \infty}\left\|W_{n+1} w_{n}-W_{n} w_{n}\right\|=0$. By the definition of $W_{n}$, it follows that

$$
\begin{aligned}
\left\|W_{n+1} w_{n}-W_{n} w_{n}\right\|= & \| \gamma_{n+1, N} S_{N} U_{n+1, N-1} w_{n}+\left(1-\gamma_{n+1, N}\right) U_{n+1, N-1} w_{n} \\
& -\gamma_{n, N} S_{N} U_{n, N-1} w_{n}-\left(1-\gamma_{n, N}\right) U_{n, N-1} w_{n} \| \\
\leq & \left\|\gamma_{n+1, N} S_{N} U_{n+1, N-1} w_{n}-\gamma_{n+1, N} S_{N} U_{n, N-1} w_{n}\right\| \\
& +\left\|\gamma_{n+1, N} S_{N} U_{n, N-1} w_{n}-\gamma_{n, N} S_{N} U_{n, N-1} w_{n}\right\| \\
& +\left\|U_{n+1, N-1} w_{n}-\gamma_{n+1, N} U_{n+1, N-1} w_{n}-U_{n, N-1} w_{n}+\gamma_{n, N} U_{n, N-1} w_{n}\right\| \\
\leq & \gamma_{n+1, N}\left\|S_{N} U_{n+1, N-1} w_{n}-S_{N} U_{n, N-1} w_{n}\right\|+\left|\gamma_{n+1, N}-\gamma_{n, N}\right|\left\|S_{N} U_{n, N-1} w_{n}\right\| \\
& +\left\|U_{n+1, N-1} w_{n}-U_{n, N-1} w_{n}\right\|+\left\|\gamma_{n+1, N} U_{n+1, N-1} w_{n}-\gamma_{n, N} U_{n, N-1} w_{n}\right\| \\
\leq & \gamma_{n+1, N}\left\|U_{n+1, N-1} w_{n}-U_{n, N-1} w_{n}\right\|+\left|\gamma_{n+1, N}-\gamma_{n, N}\right|\left\|S_{N} U_{n, N-1} w_{n}\right\| \\
& +\left\|U_{n+1, N-1} w_{n}-U_{n, N-1} w_{n}\right\|+\left\|\gamma_{n+1, N} U_{n+1, N-1} w_{n}-\gamma_{n, N} U_{n+1, N-1} w_{n}\right\| \\
& +\left\|\gamma_{n, N} U_{n+1, N-1} w_{n}-\gamma_{n, N} U_{n, N-1} w_{n}\right\| \\
\leq & \gamma_{n+1, N}\left\|U_{n+1, N-1} w_{n}-U_{n, N-1} w_{n}\right\|+\left|\gamma_{n+1, N}-\gamma_{n, N}\right|\left\|S_{N} U_{n, N-1} w_{n}\right\| \\
& +\left\|U_{n+1, N-1} w_{n}-U_{n, N-1} w_{n}\right\|+\left|\gamma_{n+1, N}-\gamma_{n, N}\right|\left\|U_{n+1, N-1} w_{n}\right\| \\
& +\gamma_{n, N}\left\|U_{n+1, N-1} w_{n}-U_{n, N-1} w_{n}\right\| \\
\leq & \left|\gamma_{n+1, N}+1+\gamma_{n, N}\right|\left\|U_{n+1, N-1} w_{n}-U_{n, N-1} w_{n}\right\|+2 M_{2}\left|\gamma_{n+1, N}-\gamma_{n, N}\right| \\
&
\end{aligned}
$$

where $M_{2}$ is an approximate constant such that $M_{2} \geq \max \left\{\sup _{n \geq 1}\left\{\left\|S_{i} U_{n, i-1} w_{n}\right\|\right\}\right.$, $\left.\sup _{n \geq 0}\left\{\left\|U_{n+1, i-1} w_{n}\right\|\right\} \mid i=1,2, \ldots\right\}$. Since $0<\gamma_{n, i} \leq 1$ for all $n \geq 1$ and $i=1,2, \ldots, N$, we compute

$$
\begin{aligned}
\left\|U_{n+1, N-1} w_{n}-U_{n, N-1} w_{n}\right\| \\
=\| \gamma_{n+1, N-1} S_{N-1} U_{n+1, N-2} w_{n}+\left(1-\gamma_{n+1, N-1}\right) U_{n+1, N-2} w_{n} \\
\quad \quad-\gamma_{n, N-1} S_{N-1} U_{n, N-2} w_{n}-\left(1-\gamma_{n, N-1}\right) U_{n, N-2} w_{n} \| \\
\leq\left\|\gamma_{n+1, N-1} S_{N-1} U_{n+1, N-2} w_{n}-\gamma_{n, N-1} S_{N-1} U_{n, N-2} w_{n}\right\| \\
\quad+\left\|U_{n+1, N-2} w_{n}-\gamma_{n+1, N-1} U_{n+1, N-2} w_{n}-U_{n, N-2} w_{n}+\gamma_{n, N-1} U_{n, N-2} w_{n}\right\| \\
\leq\left\|\gamma_{n+1, N-1} S_{N-1} U_{n+1, N-2} w_{n}-\gamma_{n+1, N-1} S_{N-1} U_{n, N-2} w_{n}\right\| \\
\quad+\left\|\gamma_{n+1, N-1} S_{N-1} U_{n, N-2} w_{n}-\gamma_{n, N-1} S_{N-1} U_{n, N-2} w_{n}\right\| \\
\quad+\left\|U_{n+1, N-2} w_{n}-U_{n, N-2} w_{n}\right\|+\left\|\gamma_{n+1, N-1} U_{n+1, N-2} w_{n}-\gamma_{n, N-1} U_{n, N-2} w_{n}\right\|
\end{aligned}
$$


Abstract and Applied Analysis

$$
\begin{aligned}
\leq & \gamma_{n+1, N-1}\left\|S_{N-1} U_{n+1, N-2} w_{n}-S_{N-1} U_{n, N-2} w_{n}\right\| \\
& +\left|\gamma_{n+1, N-1}-\gamma_{n, N-1}\right|\left\|S_{N-1} U_{n, N-2} w_{n}\right\|+\left\|U_{n+1, N-2} w_{n}-U_{n, N-2} w_{n}\right\| \\
& +\left\|\gamma_{n+1, N-1} U_{n+1, N-2} w_{n}-\gamma_{n+1, N-1} U_{n, N-2} w_{n}\right\| \\
& +\left\|\gamma_{n+1, N-1} U_{n, N-2} w_{n}-\gamma_{n, N-1} U_{n, N-2} w_{n}\right\| \\
\leq & \gamma_{n+1, N-1}\left\|U_{n+1, N-2} w_{n}-U_{n, N-2} w_{n}\right\| \\
& +\left|\gamma_{n+1, N-1}-\gamma_{n, N-1}\right|\left\|S_{N-1} U_{n, N-2} w_{n}\right\|+\left\|U_{n+1, N-2} w_{n}-U_{n, N-2} w_{n}\right\| \\
& +\gamma_{n+1, N-1}\left\|U_{n+1, N-2} w_{n}-U_{n, N-2} w_{n}\right\|+\left|\gamma_{n+1, N-1}-\gamma_{n, N-1}\right|\left\|U_{n, N-2} w_{n}\right\| \\
\leq & \left|\gamma_{n+1, N-1}+1+\gamma_{n+1, N-1}\right|\left\|U_{n+1, N-2} w_{n}-U_{n, N-2} w_{n}\right\| \\
& +2 M_{3}\left|\gamma_{n+1, N-1}-\gamma_{n, N-1}\right|,
\end{aligned}
$$

where $M_{3}$ is an approximate constant such that $M_{3} \geq \max \left\{\sup _{n \geq 1}\left\{\left\|S_{i} U_{n, i-1} w_{n}\right\|\right\}\right.$, $\left.\sup _{n \geq 0}\left\{\left\|U_{n+1, i-1} w_{n}\right\|\right\} \mid i=2,3, \ldots\right\}$. It follows that

$$
\begin{aligned}
\left\|U_{n+1, N-1} w_{n}-U_{n, N-1} w_{n}\right\| \leq & 2 M_{3}\left|\gamma_{n+1, N-1}-\gamma_{n, N-1}\right|+2 M_{3}\left|\gamma_{n+1, N-2}-\gamma_{n, N-2}\right| \\
& +\left\|U_{n+1, N-3} w_{n}-U_{n, N-3} w_{n}\right\| \\
\leq & 2 M_{3} \sum_{i=2}^{N-1}\left|\gamma_{n+1, i}-\gamma_{n, i}\right|+\left\|U_{n+1,1} w_{n}-U_{n, 1} w_{n}\right\| \\
= & 2 M_{3} \sum_{i=2}^{N-1}\left|\gamma_{n+1, i}-\gamma_{n, i}\right| \\
& +\left\|\gamma_{n+1,1} S_{1} w_{n}+\left(1-\gamma_{n+1,1}\right) w_{n}-\gamma_{n, 1} S_{1} w_{n}-\left(1-\gamma_{n, 1}\right) w_{n}\right\| \\
\leq & 2 M_{3} \sum_{i=1}^{N-1}\left|\gamma_{n+1, i}-\gamma_{n, i}\right| .
\end{aligned}
$$

Substituting (3.14) into (3.12), it yields that

$$
\begin{aligned}
\left\|W_{n+1} w_{n}-W_{n} w_{n}\right\| & \leq 2 M_{2}\left|\gamma_{n+1, N}-\gamma_{n, N}\right|+2 \gamma_{n+1, N} M_{3} \sum_{i=1}^{N-1}\left|\gamma_{n+1, i}-\gamma_{n, i}\right| \\
& \leq 2 M \sum_{i=1}^{N}\left|\gamma_{n+1, i}-\gamma_{n, i}\right|
\end{aligned}
$$

where $M$ is an approximate constant such that $M \geq \max \left\{M_{2}, M_{3}\right\}$. By condition (C3), we obtain that $\left\|W_{n+1} w_{n}-W_{n} w_{n}\right\| \rightarrow 0$ as $n \rightarrow \infty$. 
Step 5. We will show that

$$
\lim _{n \rightarrow \infty}\left\|x_{n+1}-x_{n}\right\|=0
$$

We observe that

$$
\begin{aligned}
\left\|x_{n+1}-x_{n}\right\|= & \left\|\alpha_{n} \gamma \Phi\left(x_{n}\right)+\left(I-\alpha_{n} \mu B\right) w_{n}-\alpha_{n-1} \gamma \Phi\left(x_{n-1}\right)-\left(I-\alpha_{n-1} \mu B\right) w_{n-1}\right\| \\
\leq & \left\|\alpha_{n} \gamma\left(\Phi\left(x_{n}\right)-\Phi\left(x_{n-1}\right)\right)+\left(I-\alpha_{n} \mu B\right) w_{n}-\left(I-\alpha_{n} \mu B\right) w_{n-1}\right\| \\
& +\left\|\gamma\left(\alpha_{n}-\alpha_{n-1}\right) \Phi\left(x_{n-1}\right)+\mu\left(\alpha_{n}-\alpha_{n-1}\right) B w_{n-1}\right\| \\
\leq & \alpha_{n} \gamma\left\{\left\|x_{n}-x_{n-1}\right\|-\pi\left(\left\|x_{n}-x_{n+1}\right\|\right)\right\}+\left(1-\alpha_{n} \tau\right)\left\|w_{n}-w_{n-1}\right\|+2 M_{4}\left|\alpha_{n}-\alpha_{n-1}\right| \\
\leq & \alpha_{n} \gamma\left\|x_{n}-x_{n-1}\right\|+\left(1-\alpha_{n} \tau\right)\left\|w_{n}-w_{n-1}\right\|+2 M_{4}\left|\alpha_{n}-\alpha_{n-1}\right|,
\end{aligned}
$$

where $M_{4}$ is an approximate constant such that $M_{4} \geq \max \left\{\sup _{n \geq 2}\left\{\left\|\Phi\left(x_{n-1}\right)\right\|\right\}\right.$, $\left.\sup _{n \geq 2}\left\{\left\|B w_{n-1}\right\|\right\}\right\}$. We compute

$$
\begin{aligned}
\left\|w_{n}-w_{n-1}\right\|= & \left\|W_{n} \Im_{n}^{M} x_{n}-W_{n-1} \Im_{n-1}^{M} x_{n-1}\right\| \\
\leq & \left\|W_{n} \Im_{n}^{M} x_{n}-W_{n} \Im_{n}^{M} x_{n-1}\right\|+\left\|W_{n} \Im_{n}^{M} x_{n-1}-W_{n} \Im_{n-1}^{M} x_{n-1}\right\| \\
& +\left\|W_{n} \Im_{n-1}^{M} x_{n-1}-W_{n-1} \Im_{n-1}^{M} x_{n-1}\right\| \\
\leq & \left\|x_{n}-x_{n-1}\right\|+\left\|\Im_{n}^{M} x_{n-1}-\Im_{n-1}^{M} x_{n-1}\right\| \\
& +\left\|W_{n} \Im_{n-1}^{M} x_{n-1}-W_{n-1} \Im_{n-1}^{M} x_{n-1}\right\| .
\end{aligned}
$$

By Step 2 and Step 4, we have immediately concluded from (3.17) that

$$
\begin{aligned}
\left\|x_{n+1}-x_{n}\right\| & \leq \alpha_{n} \gamma\left\|x_{n}-x_{n-1}\right\|+\left(1-\alpha_{n} \tau\right)\left\|w_{n}-w_{n-1}\right\|+2 M_{4}\left|\alpha_{n}-\alpha_{n-1}\right| \\
& \leq \alpha_{n} \gamma\left\|x_{n}-x_{n-1}\right\|+\left(1-\alpha_{n} \tau\right)\left\|x_{n}-x_{n-1}\right\|+2 M_{4}\left|\alpha_{n}-\alpha_{n-1}\right| \\
& \leq\left(1-\alpha_{n}(\tau-\gamma)\right)\left\|x_{n}-x_{n-1}\right\|+2 M_{4}\left|\alpha_{n}-\alpha_{n-1}\right| .
\end{aligned}
$$

By Lemma 2.5, we have $\lim _{n \rightarrow \infty}\left\|x_{n+1}-x_{n}\right\|=0$.

Step 6. We will show that

$$
\lim _{n \rightarrow \infty}\left\|\Im_{n}^{k} x_{n}-\Im_{n}^{k+1} x_{n}\right\|=0, \quad \forall k=1,2, \ldots, M-1
$$


For any $p \in \Theta$ and for all $k=1,2, \ldots, M-1$, note that $J_{r_{k+1, n}}^{F_{k+1}}$ is firmly nonexpansive. Then by Lemma 2.11, we get

$$
\begin{aligned}
\left\|\Im_{n}^{k+1} x_{n}-p\right\|^{2} & =\left\|J_{r_{k+1, n}}^{F_{k+1}} \Im_{n}^{k} x_{n}-J_{r_{k+1, n}}^{F_{k+1}} p\right\|^{2} \\
& \leq\left\langle J_{r_{k+1, n}}^{F_{k+1}} \Im_{n}^{k} x_{n}-J_{r_{k+1, n}}^{F_{k+1}} p, \Im_{n}^{k} x_{n}-p\right\rangle \\
& =\left\langle\Im_{n}^{k+1} x_{n}-p, \Im_{n}^{k} x_{n}-p\right\rangle \\
& =\frac{1}{2}\left(\left\|\Im_{n}^{k+1} x_{n}-p\right\|^{2}+\left\|\Im_{n}^{k} x_{n}-p\right\|^{2}-\left\|\Im_{n}^{k+1} x_{n}-\Im_{n}^{k} x_{n}\right\|^{2}\right),
\end{aligned}
$$

and, hence,

$$
\begin{aligned}
\left\|\Im_{n}^{k+1} x_{n}-p\right\|^{2} & \leq\left\|\Im_{n}^{k} x_{n}-p\right\|^{2}-\left\|\Im_{n}^{k+1} x_{n}-\Im_{n}^{k} x_{n}\right\|^{2} \\
& \leq\left\|x_{n}-p\right\|^{2}-\left\|\Im_{n}^{k+1} x_{n}-\Im_{n}^{k} x_{n}\right\|^{2} .
\end{aligned}
$$

By (3.22), we compute

$$
\begin{aligned}
\left\|x_{n+1}-p\right\|^{2}= & \left\|\alpha_{n} \gamma \Phi\left(x_{n}\right)+\left(I-\alpha_{n} \mu B\right) w_{n}-p\right\|^{2} \\
= & \left\|\alpha_{n} \gamma \Phi\left(x_{n}\right)+\left(I-\alpha_{n} \mu B\right) W_{n} \Im_{n}^{M} x_{n}-p\right\|^{2} \\
= & \left\|\alpha_{n}\left(\gamma \Phi\left(x_{n}\right)-\mu B p\right)+\left(I-\alpha_{n} \mu B\right) W_{n} \Im_{n}^{M} x_{n}-\left(I-\alpha_{n} \mu B\right) p\right\|^{2} \\
= & \alpha_{n}^{2}\left\|\gamma \Phi\left(x_{n}\right)-\mu B p\right\|^{2}+\left\|\left(I-\alpha_{n} \mu B\right) W_{n} \Im_{n}^{M} x_{n}-\left(I-\alpha_{n} \mu B\right) W_{n} \Im_{n}^{M} p\right\|^{2} \\
& +2 \alpha_{n}\left\langle\gamma \Phi\left(x_{n}\right)-\mu B p,\left(I-\alpha_{n} \mu B\right) W_{n} \Im_{n}^{M} x_{n}-\left(I-\alpha_{n} \mu B\right) W_{n} \Im_{n}^{M} p\right\rangle \\
\leq & \alpha_{n}^{2}\left\|\gamma \Phi\left(x_{n}\right)-\mu B p\right\|^{2}+\left(1-\alpha_{n} \tau\right)^{2}\left\|\Im_{n}^{M} x_{n}-\Im_{n}^{M} p\right\|^{2} \\
& +2 \alpha_{n}\left\langle\gamma \Phi\left(x_{n}\right)-\mu B p, W_{n} \Im_{n}^{M} x_{n}-W_{n} \Im_{n}^{M} p\right\rangle \\
& +2 \alpha_{n}^{2} \mu\left\langle\gamma \Phi\left(x_{n}\right)-\mu B p, B W_{n} \Im_{n}^{M} x_{n}-B W_{n} \Im_{n}^{M} p\right\rangle \\
\leq & \alpha_{n}^{2}\left\|\gamma \Phi\left(x_{n}\right)-\mu B p\right\|^{2}+\left(1-\alpha_{n} \tau\right)^{2}\left\|\Im_{n}^{M} x_{n}-p\right\|^{2} \\
& +2 \alpha_{n}\left\langle\gamma \Phi\left(x_{n}\right)-\gamma \Phi(p), W_{n} \Im_{n}^{M} x_{n}-W_{n} \Im_{n}^{M} p\right\rangle \\
& +2 \alpha_{n}\left\langle\gamma \Phi(p)-\mu B(p), W_{n} \Im_{n}^{M} x_{n}-W_{n} \Im_{n}^{M} p\right\rangle \\
& +2 \alpha_{n}^{2} \mu\left\|\gamma \Phi\left(x_{n}\right)-\mu B p\right\|\left\|B W_{n} \Im_{n}^{M} x_{n}-B W_{n} \Im_{n}^{M} p\right\|
\end{aligned}
$$




$$
\begin{aligned}
& \leq \alpha_{n}^{2}\left\|\gamma \Phi\left(x_{n}\right)-\mu B p\right\|^{2}+\left(1-\alpha_{n} \tau\right)^{2}\left\|\Im_{n}^{M} x_{n}-p\right\|^{2} \\
& +2 \alpha_{n} \gamma\left\{\left\|x_{n}-p\right\|-\pi\left(\left\|x_{n}-p\right\|\right)\right\}\left\|x_{n}-p\right\| \\
& +2 \alpha_{n}\left\langle\gamma \Phi(p)-\mu B(p), W_{n} \Im_{n}^{M} x_{n}-W_{n} \Im_{n}^{M} p\right\rangle \\
& +2 \alpha_{n}^{2} \mu\left\|\gamma \Phi\left(x_{n}\right)-\mu B p\right\|\left\|B W_{n} \Im_{n}^{M} x_{n}-B W_{n} \Im_{n}^{M} p\right\| \\
& \leq \alpha_{n}^{2}\left\|\gamma \Phi\left(x_{n}\right)-\mu B p\right\|^{2}+\left(1-2 \alpha_{n} \tau+\alpha_{n}^{2} \tau^{2}+2 \alpha_{n} \gamma\right)\left\|\Im_{n}^{M} x_{n}-p\right\|^{2} \\
& +2 \alpha_{n}\left\langle\gamma \Phi(p)-\mu B(p), W_{n} \Im_{n}^{M} x_{n}-W_{n} \Im_{n}^{M} p\right\rangle \\
& +2 \alpha_{n}^{2} \mu\left\|\gamma \Phi\left(x_{n}\right)-\mu B p\right\|\left\|B W_{n} \Im_{n}^{M} x_{n}-B W_{n} \Im_{n}^{M} p\right\| \\
& \leq\left(1-2 \alpha_{n}(\tau-\gamma)\right)\left\|\Im_{n}^{k+1} x_{n}-p\right\|^{2} \\
& +\alpha_{n}\left(\alpha_{n} \tau^{2}\left\|\Im_{n}^{M} x_{n}-p\right\|^{2}+\alpha_{n}\left\|\gamma \Phi\left(x_{n}\right)-\mu B p\right\|^{2}\right. \\
& +2\left\langle\gamma \Phi(p)-\mu B(p), W_{n} \Im_{n}^{M} x_{n}-W_{n} \Im_{n}^{M} p\right\rangle \\
& \left.+2 \alpha_{n} \mu\left\|\gamma \Phi\left(x_{n}\right)-\mu B p\right\|\left\|B W_{n} \Im_{n}^{M} x_{n}-B W_{n} \Im_{n}^{M} p\right\|\right) \\
& \leq\left(1-2 \alpha_{n}(\tau-\gamma)\right)\left(\left\|x_{n}-p\right\|^{2}-\left\|\Im_{n}^{k} x_{n}-\Im_{n}^{k+1} x_{n}\right\|^{2}\right) \\
& +\alpha_{n}\left(\alpha_{n} \tau^{2}\left\|x_{n}-p\right\|^{2}+\alpha_{n}\left\|\gamma \Phi\left(x_{n}\right)-\mu B p\right\|^{2}\right. \\
& +2\left\langle\gamma \Phi(p)-\mu B(p), W_{n} \Im_{n}^{M} x_{n}-W_{n} \Im_{n}^{M} p\right\rangle \\
& \left.+2 \alpha_{n} \mu\left\|\gamma \Phi\left(x_{n}\right)-\mu B p\right\|\left\|B W_{n} \Im_{n}^{M} x_{n}-B W_{n} \Im_{n}^{M} p\right\|\right) .
\end{aligned}
$$

So, we obtain

$$
\begin{aligned}
\left(1-2 \alpha_{n}(\tau-\gamma)\right)\left\|\Im_{n}^{k} x_{n}-\Im_{n}^{k+1} x_{n}\right\|^{2} \leq & \left(1-2 \alpha_{n}(\tau-\gamma)\right)\left\|x_{n}-p\right\|^{2}-\left\|x_{n+1}-p\right\|^{2} \\
& +\alpha_{n}\left(\alpha_{n} \tau^{2}\left\|x_{n}-p\right\|^{2}+\alpha_{n}\left\|\gamma \Phi\left(x_{n}\right)-\mu B p\right\|^{2}\right. \\
& +2\left\langle\gamma \Phi(p)-\mu B(p), W_{n} \Im_{n}^{M} x_{n}-W_{n} \Im_{n}^{M} p\right\rangle \\
& \left.+2 \alpha_{n} \mu\left\|\gamma \Phi\left(x_{n}\right)-\mu B p\right\|\left\|B W_{n} \Im_{n}^{M} x_{n}-B W_{n} \Im_{n}^{M} p\right\|\right)
\end{aligned}
$$


Abstract and Applied Analysis

$$
\begin{aligned}
\leq \| x_{n}- & x_{n+1} \|\left(\left\|x_{n}-p\right\|+\left\|x_{n+1}-p\right\|\right) \\
+\alpha_{n} & \left(\alpha_{n} \tau^{2}\left\|x_{n}-p\right\|^{2}+\alpha_{n}\left\|\gamma \Phi\left(x_{n}\right)-\mu B p\right\|^{2}\right. \\
& +2\left\langle\gamma \Phi(p)-\mu B(p), W_{n} \Im_{n}^{M} x_{n}-W_{n} \Im_{n}^{M} p\right\rangle \\
& \left.+2 \alpha_{n} \mu\left\|\gamma \Phi\left(x_{n}\right)-\mu B p\right\|\left\|B W_{n} \Im_{n}^{M} x_{n}-B W_{n} \Im_{n}^{M} p\right\|\right) .
\end{aligned}
$$

Using condition (C1) and (3.16), we obtain

$$
\lim _{n \rightarrow \infty}\left\|\Im_{n}^{k} x_{n}-\Im_{n}^{k+1} x_{n}\right\|=0, \quad \forall k=1,2, \ldots, M-1
$$

Step 7. Next, we show that

$$
\lim _{n \rightarrow \infty}\left\|x_{n}-W_{n} \Im_{n}^{M} x_{n}\right\|=0
$$

Since

$$
\begin{aligned}
\left\|x_{n}-W_{n} \Im_{n}^{M} x_{n}\right\| & \leq\left\|x_{n}-x_{n+1}\right\|+\left\|x_{n+1}-W_{n} \Im_{n}^{M} x_{n}\right\| \\
& =\left\|x_{n}-x_{n+1}\right\|+\alpha_{n}\left\|\gamma f\left(x_{n}\right)-\mu B W_{n} \Im_{n}^{M} x_{n}\right\| \\
& \leq\left\|x_{n}-x_{n+1}\right\|+\alpha_{n}\left\|\gamma f\left(x_{n}\right)-\mu B x_{n}\right\|,
\end{aligned}
$$

by condition (C1) and (3.16), we get $\left\|x_{n}-W_{n} \Im_{n}^{M} x_{n}\right\| \rightarrow 0$ as $n \rightarrow \infty$.

Step 8. We show that $z \in \Theta$. The weak $w$-limit set of $\left\{x_{n}\right\}, w_{w}\left(x_{n}\right)$ is a subset of $\Theta$. Let $z \in$ $w_{w}\left(x_{n}\right)$, and let $\left\{x_{n_{m}}\right\}$ be a subsequence of $\left\{x_{n}\right\}$ which converges weakly to $z$. By Step 6, without loss of generality, we may assume that

$$
\Im_{n_{m}}^{k} x_{n_{m}} \rightarrow z, \quad \forall k \in\{1,2, \ldots, M-1\}
$$

We need to show that $z \in \Theta$. At first, note that by (A2) and given $y \in C$ and $k \in\{1,2, \ldots, M-$ $1\}$, we have

$$
\frac{1}{r_{k+1, n}}\left\langle y-\Im_{n}^{k+1} x_{n}, \Im_{n}^{k+1} x_{n}-\Im_{n}^{k} x_{n}\right\rangle \geq F_{k+1}\left(y, \Im_{n}^{k+1} x_{n}\right) .
$$

Thus,

$$
\left\langle y-\Im_{n_{m}}^{k+1} x_{n_{m}}, \frac{\Im_{n_{m}}^{k+1} x_{n_{m}}-\Im_{n_{m}}^{k} x_{n_{m}}}{r_{k+1, n_{m}}}\right\rangle \geq F_{k+1}\left(y, \Im_{n_{m}}^{k+1} x_{n_{m}}\right) .
$$


By $(\mathrm{A} 4), F(y, \cdot)$ is a lower semicontinuous and convex, thus, weakly semicontinuous. By condition (C3) and (3.20), imply that

$$
\frac{\Im_{n_{m}}^{k+1} x_{n_{m}}-\Im_{n_{m}}^{k} x_{n_{m}}}{r_{k+1, n_{m}}} \longrightarrow 0,
$$

in norm. Therefore, letting $m \rightarrow \infty$ in (3.30) yields

$$
F_{k+1}(y, z) \leq \lim _{m \rightarrow \infty} F_{k+1}\left(y, \Im_{n_{m}}^{k+1} x_{n_{m}}\right) \leq 0
$$

for all $y \in H$ and $k \in\{1,2, \ldots, M-1\}$. Replacing $y$ with $y_{t}=t y+(1-t) z$ with $t \in(0,1)$ and using (A1) and (A4), we obtain

$$
0=F_{k+1}\left(y_{t}, y_{t}\right) \leq t F_{k+1}\left(y_{t}, y\right)+(1-t) F_{k+1}\left(y_{t}, z\right) \leq t F_{k+1}\left(y_{t}, y\right)
$$

Hence, $F_{k+1}(t y+(1-t) z, y) \geq 0$, for all $t \in(0,1)$ and $y \in H$. Letting $t \rightarrow 0^{+}$and using (A3), we conclude $F_{k+1}(z, y) \geq 0$, for all $y \in H$ and $k \in\{1,2, \ldots, M\}$. Therefore,

$$
z \in \bigcap_{j=1}^{M} \operatorname{EP}\left(F_{j}\right)=\operatorname{EP}(\mathcal{F}) .
$$

Next, we show that $z \in \bigcap_{i=1}^{\infty} \operatorname{EP}\left(S_{i}\right)$. By Lemma 2.12, we have for all $z \in C$,

$$
W_{n_{m}} z \longrightarrow W z
$$

and $F(W)=\bigcap_{i=1}^{\infty} F\left(S_{i}\right)$. Assume that $z \notin F(W)$, then $z \neq W z$. Therefore, from Opial's property of Hilbert space, (3.26), (3.34), and (3.35), we have

$$
\begin{aligned}
\liminf _{m \rightarrow \infty}\left\|x_{n_{m}}-z\right\| & <\liminf _{m \rightarrow \infty}\left\|x_{n_{m}}-W z\right\| \\
& \leq \liminf _{m \rightarrow \infty}\left\{\left\|x_{n_{m}}-W_{n_{m}} \Im_{n_{m}}^{M} x_{n_{m}}\right\|+\left\|W_{n_{m}} \Im_{n_{m}}^{M} x_{n_{m}}-W_{n_{m}} z\right\|+\left\|W_{n_{m}} z-W z\right\|\right\} \\
& \leq \liminf _{m \rightarrow \infty}\left\{\left\|x_{n_{m}}-z\right\|+\left\|W_{n_{m}} z-W z\right\|\right\} \\
& \leq \liminf _{m \rightarrow \infty}\left\|x_{n_{m}}-z\right\| .
\end{aligned}
$$

This is a contradiction. Therefore, $z$ must belong to $F(W)=\bigcap_{i=1}^{\infty} F\left(S_{i}\right)$.

Step 9. We show that $\limsup _{n \rightarrow \infty}\left\langle(\mu B-\gamma \Phi) x^{*}, x^{*}-x_{n}\right\rangle \leq 0$, where $x^{*}=P_{\Theta}(I-\mu B+\gamma \Phi) x^{*}$. By Banach's contraction mapping principle, it guarantees that $P_{\Theta}(I-\mu B+\gamma \Phi)$ has a unique 
fixed point $x^{*}$ which is the unique solution of (3.2). Let $\left\{x_{n_{k}}\right\}$ be a subsequence of $\left\{x_{n}\right\}$ such that

$$
\lim _{k \rightarrow \infty}\left\langle(\mu B-\gamma \Phi) x^{*}, x^{*}-x_{n_{k}}\right\rangle=\limsup _{n \rightarrow \infty}\left\langle(\mu B-\gamma \Phi) x^{*}, x^{*}-x_{n}\right\rangle
$$

Without loss of generality, we can assume that $\left\{x_{n_{k}}\right\}$ converges weakly to some $z \in C$. It follows that from Lemma 2.6 and $\lim _{n \rightarrow \infty}\left\|x_{n+1}-x_{n}\right\|=0$ that $z \in \Theta$. Hence by (3.2), we obtain

$$
\limsup _{n \rightarrow \infty}\left\langle(\mu B-\gamma \Phi) x^{*}, x^{*}-x_{n}\right\rangle=\left\langle(\mu B-\gamma \Phi) x^{*}, x^{*}-z\right\rangle \leq 0
$$

Step 10. Finally, we show that $x_{n} \rightarrow x^{*}$. As a matter of fact, we have

$$
\begin{aligned}
\left\|x_{n+1}-x^{*}\right\|^{2}= & \left\|\alpha_{n} \gamma \Phi\left(x_{n}\right)+\left(I-\alpha_{n} \mu B\right) w_{n}-x^{*}\right\|^{2} \\
= & \left\|\alpha_{n} \gamma \Phi\left(x_{n}\right)+\left(I-\alpha_{n} \mu B\right) W_{n} \Im_{n}^{M} x_{n}-x^{*}\right\|^{2} \\
= & \left\|\alpha_{n}\left(\gamma \Phi\left(x_{n}\right)-\mu B x^{*}\right)+\left(I-\alpha_{n} \mu B\right) W_{n} \Im_{n}^{M} x_{n}-\left(I-\alpha_{n} \mu B\right) x^{*}\right\|^{2} \\
= & \alpha_{n}^{2}\left\|\gamma \Phi\left(x_{n}\right)-\mu B x^{*}\right\|^{2}+\left\|\left(I-\alpha_{n} \mu B\right) W_{n} \Im_{n}^{M} x_{n}-\left(I-\alpha_{n} \mu B\right) W_{n} \Im_{n}^{M} x^{*}\right\|^{2} \\
& +2 \alpha_{n}\left\langle\gamma \Phi\left(x_{n}\right)-\mu B x^{*},\left(I-\alpha_{n} \mu B\right) W_{n} \Im_{n}^{M} x_{n}-\left(I-\alpha_{n} \mu B\right) W_{n} \Im_{n}^{M} x^{*}\right\rangle \\
\leq & \alpha_{n}^{2}\left\|\gamma \Phi\left(x_{n}\right)-\mu B x^{*}\right\|^{2}+\left(1-\alpha_{n} \tau\right)^{2}\left\|x_{n}-x^{*}\right\|^{2} \\
& +2 \alpha_{n}\left\langle\gamma \Phi\left(x_{n}\right)-\mu B x^{*}, W_{n} \Im_{n}^{M} x_{n}-W_{n} \Im_{n}^{M} x^{*}\right\rangle \\
& -2 \alpha_{n}^{2} \mu\left\langle\gamma \Phi\left(x_{n}\right)-\mu B x^{*}, B W_{n} \Im_{n}^{M} x_{n}-B W_{n} \Im_{n}^{M} x^{*}\right\rangle \\
\leq & \alpha_{n}^{2}\left\|\gamma \Phi\left(x_{n}\right)-\mu B x^{*}\right\|^{2}+\left(1-\alpha_{n} \tau\right)^{2}\left\|x_{n}-x^{*}\right\|^{2} \\
& +2 \alpha_{n}\left\langle\gamma \Phi\left(x_{n}\right)-\gamma \Phi\left(x^{*}\right), W_{n} \Im_{n}^{M} x_{n}-W_{n} \Im_{n}^{M} x^{*}\right\rangle \\
& +2 \alpha_{n}\left\langle\gamma \Phi\left(x^{*}\right)-\mu B\left(x^{*}\right), W_{n} \Im_{n}^{M} x_{n}-W_{n} \Im_{n}^{M} x^{*}\right\rangle \\
& -2 \alpha_{n}^{2} \mu\left\|\gamma \Phi\left(x_{n}\right)-\mu B x^{*}\right\|\left\|B W_{n} \Im_{n}^{M} x_{n}-B W_{n} \Im_{n}^{M} x^{*}\right\| \\
\leq & \alpha_{n}^{2}\left\|\gamma \Phi\left(x_{n}\right)-\mu B x^{*}\right\|^{2}+\left(1-\alpha_{n} \tau\right)^{2}\left\|x_{n}-x^{*}\right\|^{2}
\end{aligned}
$$




$$
\begin{aligned}
& +2 \alpha_{n} \gamma\left\{\left\|x_{n}-x^{*}\right\|-\pi\left(\left\|x_{n}-x^{*}\right\|\right)\right\}\left\|x_{n}-x^{*}\right\| \\
& +2 \alpha_{n}\left\langle\gamma \Phi\left(x^{*}\right)-\mu B\left(x^{*}\right), W_{n} \Im_{n}^{M} x_{n}-W_{n} \Im_{n}^{M} x^{*}\right\rangle \\
& +2 \alpha_{n}^{2} \mu\left\|\gamma \Phi\left(x_{n}\right)-\mu B x^{*}\right\|\left\|B W_{n} \Im_{n}^{M} x_{n}-B W_{n} \Im_{n}^{M} x^{*}\right\| \\
& \leq \alpha_{n}^{2}\left\|\gamma \Phi\left(x_{n}\right)-\mu B x^{*}\right\|^{2}+\left(1-2 \alpha_{n} \tau+\alpha_{n}^{2} \tau^{2}+2 \alpha_{n} \gamma\right)\left\|x_{n}-x^{*}\right\|^{2} \\
& +2 \alpha_{n}\left\langle\gamma \Phi\left(x^{*}\right)-\mu B\left(x^{*}\right), W_{n} \Im_{n}^{M} x_{n}-W_{n} \Im_{n}^{M} x^{*}\right\rangle \\
& +2 \alpha_{n}^{2} \mu\left\|\gamma \Phi\left(x_{n}\right)-\mu B x^{*}\right\|\left\|B W_{n} \Im_{n}^{M} x_{n}-B W_{n} \Im_{n}^{M} x^{*}\right\| \\
& \leq\left(1-2 \alpha_{n}(\tau-\gamma)\right)\left\|x_{n}-x^{*}\right\|^{2} \\
& +\alpha_{n}\left(\alpha_{n} \tau^{2}\left\|x_{n}-x^{*}\right\|^{2}+\alpha_{n}\left\|\gamma \Phi\left(x_{n}\right)-\mu B x^{*}\right\|^{2}\right. \\
& +2\left\langle\gamma \Phi\left(x^{*}\right)-\mu B\left(x^{*}\right), W_{n} \Im_{n}^{M} x_{n}-W_{n} \Im_{n}^{M} x^{*}\right\rangle \\
& \left.+2 \alpha_{n} \mu\left\|\gamma \Phi\left(x_{n}\right)-\mu B x^{*}\right\|\left\|B W_{n} \Im_{n}^{M} x_{n}-B W_{n} \Im_{n}^{M} x^{*}\right\|\right),
\end{aligned}
$$

where

$$
\begin{gathered}
l_{n}=2 \alpha_{n}(\tau-\gamma), \\
\delta_{n}=\frac{1}{2(\tau-\gamma)}\left(\alpha_{n} \tau^{2}\left\|x_{n}-x^{*}\right\|^{2}+\alpha_{n}\left\|\gamma \Phi\left(x_{n}\right)-\mu B x^{*}\right\|^{2}\right. \\
+2\left\langle\gamma \Phi\left(x^{*}\right)-\mu B\left(x^{*}\right), W_{n} \Im_{n}^{M} x_{n}-W_{n} \Im_{n}^{M} x^{*}\right\rangle \\
\left.+2 \alpha_{n} \mu\left\|\gamma \Phi\left(x_{n}\right)-\mu B x^{*}\right\|\left\|B W_{n} \Im_{n}^{M} x_{n}-B W_{n} \Im_{n}^{M} x^{*}\right\|\right) .
\end{gathered}
$$

It is easily to see that $\lim _{n \rightarrow \infty} l_{n}=0, \sum_{n=1}^{\infty} l_{n}=\infty$ and $\limsup _{n \rightarrow \infty} \delta_{n} \leq 0$. By Lemma 2.5, we conclude that $x_{n} \rightarrow x^{*}$; this completes the proof.

Corollary 3.2. Let $C$ be a nonempty closed convex subset of a real Hilbert space $H$ such that $C \pm C \subset C$. Let $\mathcal{S}=\left\{S_{i}: C \rightarrow C\right\}$ a family of infinitely nonexpansive mappings, and let $\mathcal{F}=\left\{F_{j}: j=1,2,3, \ldots, M\right\}$ be a finite family of bifunctions $C \times C$ to $\mathbb{R}$ satisfying (A1)-(A4). Assume that $\Theta:=\left(\bigcap_{i=1}^{\infty} F\left(S_{i}\right)\right) \cap\left(\bigcap_{j=1}^{M} \mathrm{EP}\left(F_{j}\right)\right) \neq \emptyset$. Let $B$ be a $k$-Lipschitzian and $\eta$-strongly monotone mapping on $C$ with $k>0, \eta>0$. Let $\Phi$ be a $\pi$-weakly contractive self-mapping on $C$ with $\alpha \in[0,1)$. Denote by $C$ the collection of all weakly contractive mapping $\Phi$ on $C$. 
Let $0<\mu<2 \eta / k^{2}$ and $0<\gamma<\mu\left(\eta-\mu k^{2} / 2\right)=\tau$. Let the mapping $W_{n}$ be defined by (2.14) and $\left\{r_{j, n}\right\}_{j=1}^{M}$ be a sequence in $(0, \infty)$. If $\left\{x_{n}\right\}$ is the sequence generated by $x_{1} \in C$ and

$$
\begin{gathered}
w_{n}=W_{n} J_{r_{M}, n}^{F_{M}} \cdots J_{r_{2}, n}^{F_{2}} J_{r_{1}, n}^{F_{1}} x_{n}, \\
x_{n+1}=\alpha_{n} \Phi\left(x_{n}\right)+\left(I-\alpha_{n} \mu B\right) w_{n}, \quad \forall n \in \mathbb{N},
\end{gathered}
$$

where $\left\{\alpha_{n}\right\}$ is a sequence in $(0,1)$ which satisfies the following conditions $(C 1)-(C 4)$, then the sequence $\left\{x_{n}\right\}$ converges strongly to $x^{*}=P_{\Theta}(I-\mu B+\Phi)\left(x^{*}\right)$ which is the unique solution of the variational inequality

$$
\left\langle(\mu B-\Phi) x^{*}, x^{*}-x\right\rangle \leq 0, \quad \forall x \in \Theta,
$$

which is the optimality condition for the minimization problem

$$
\min _{x \in F(T)} \frac{1}{2}\langle B x, x\rangle-h(x),
$$

where $h$ is a potential function for $\gamma \Phi$ (i.e., $h^{\prime}(x)=\gamma \Phi(x)$, for $x \in H$ ).

Proof. Taking $\gamma \equiv 1$, in Theorem 3.1, it is easy to obtain the desired conclusion.

Corollary 3.3. Let $C$ be a nonempty closed convex subset of a real Hilbert space $H$ such that $C \pm$ $C \subset C$. Let $\mathcal{S}=\left\{S_{i}: C \rightarrow C\right\}$ a family of infinitely nonexpansive mappings, and let $\mathcal{F}=\left\{F_{j}\right.$ : $j=1,2,3, \ldots, M\}$ be a finite family of bifunctions $C \times C$ to $\mathbb{R}$ satisfying (A1)-(A4). Assume that $\Theta:=\left(\bigcap_{i=1}^{\infty} F\left(S_{i}\right)\right) \cap\left(\bigcap_{j=1}^{M} \operatorname{EP}\left(F_{j}\right)\right) \neq \emptyset$. Let $B$ be a $k$-Lipschitzian and $\eta$-strongly monotone mapping on $C$, and let $f$ be a contraction self-mapping on $C$ with $\alpha \in[0,1)$. Denote by $C$ the collection of all contraction $f$ on $C$. Let $0<\mu<2 \eta / k^{2}$ and $0<\gamma<\mu\left(\eta-\mu k^{2} / 2\right) / \alpha=\tau / \alpha$. Let the mapping $W_{n}$ be defined by (2.14) and $\left\{r_{j, n}\right\}_{j=1}^{M}$ be a sequence in $(0, \infty)$. If $\left\{x_{n}\right\}$ is the sequence generated by $x_{1} \in C$ and

$$
\begin{gathered}
w_{n}=W_{n} J_{r_{M}, n}^{F_{M}} \cdots J_{r_{2}, n}^{F_{2}} J_{r_{1}, n}^{F_{1}} x_{n}, \\
x_{n+1}=\alpha_{n} \gamma f\left(x_{n}\right)+\left(I-\alpha_{n} \mu B\right) w_{n}, \quad \forall n \in \mathbb{N},
\end{gathered}
$$

where $\left\{\alpha_{n}\right\}$ is a sequence in $(0,1)$ which satisfies the following conditions $(C 1)-(C 4)$, then the sequence $\left\{x_{n}\right\}$ converges strongly to $x^{*}=P_{\Theta}(I-\mu B+\gamma f)\left(x^{*}\right)$, which is the unique solution of the variational inequality

$$
\left\langle(\mu B-\gamma f) x^{*}, x^{*}-x\right\rangle \leq 0, \quad \forall x \in \Theta,
$$

which is the optimality condition for the minimization problem

$$
\min _{x \in F(T)} \frac{1}{2}\langle B x, x\rangle-h(x),
$$

where $h$ is a potential function for $\gamma f\left(\right.$ i.e., $h^{\prime}(x)=\gamma f(x)$, for $x \in H$ ). 
Proof. Taking $\Phi \equiv f$ in Theorem 3.1, it is easy to obtain the desired conclusion.

Corollary 3.4. Let $C$ be a nonempty closed convex subset of a real Hilbert space $H$ such that $C \pm C \subset$ $C$, and let $\mathcal{F}=\left\{F_{j}: j=1,2,3, \ldots, M\right\}$ be a finite family of bifunctions $C \times C$ to $\mathbb{R}$ satisfying (A1)(A4). Assume that $\Theta:=\bigcap_{j=1}^{M} \mathrm{EP}\left(F_{j}\right) \neq \emptyset$. Let $B$ be a $k$-Lipschitzian and $\eta$-strongly monotone mapping on $C$ with $k>0, \eta>0$. Let $\Phi$ be a $\pi$-weakly contractive self-mapping on $C$ with $\alpha \in[0,1)$. Denote by $C$ the collection of all weakly contractivemapping on $C$, and let $f \in C$ with $\alpha=1$. Let $0<\mu<2 \eta / k^{2}$ and $0<\gamma<\mu\left(\eta-\mu k^{2} / 2\right)=\tau$. Let $\left\{r_{j, n}\right\}_{j=1}^{M}$ be a sequence in $(0, \infty)$. If $\left\{x_{n}\right\}$ is the sequence generated by $x_{1} \in C$ and

$$
\begin{gathered}
w_{n}=J_{r_{M}, n}^{F_{M}} \cdots J_{r_{2}, n}^{F_{2}} J_{r_{1}, n}^{F_{1}} x_{n}, \\
x_{n+1}=\alpha_{n} \gamma \Phi\left(x_{n}\right)+\left(I-\alpha_{n} \mu B\right) w_{n}, \quad \forall n \in \mathbb{N},
\end{gathered}
$$

where $\left\{\alpha_{n}\right\}$ is a sequence in $(0,1)$ which satisfies the following conditions $(C 1),(C 2)$, and (C4) in Theorem 3.1, then the sequence $\left\{x_{n}\right\}$ converges strongly to $x^{*}=P_{\Theta}(I-\mu B+\gamma \Phi)\left(x^{*}\right)$, which is the unique solution of the variational inequality

$$
\left\langle(\mu B-\gamma \Phi) x^{*}, x^{*}-x\right\rangle \leq 0, \quad \forall x \in \Theta
$$

which is the optimality condition for the minimization problem

$$
\min _{x \in F(T)} \frac{1}{2}\langle B x, x\rangle-h(x)
$$

where $h$ is a potential function for $\gamma \Phi$ (i.e., $h^{\prime}(x)=\gamma \Phi(x)$, for $x \in H$ ).

Proof. Taking $W_{n} \equiv 0$ in Theorem 3.1, it is easy to obtain the desired conclusion.

Corollary 3.5. Let $C$ be a nonempty closed convex subset of a real Hilbert space $H$ such that $C \pm C \subset$ $C$, and let $\mathcal{S}=\left\{S_{i}: C \rightarrow C\right\}$ a family of infinitely nonexpansive mappings. Assume that $\Theta:=$ $\bigcap_{i=1}^{\infty} F\left(S_{i}\right) \neq \emptyset$. Let $B$ be a $k$-Lipschitzian and $\eta$-strongly monotone mapping on $C$ with $k>0, \eta>0$. Let $\Phi$ be a $\pi$-weakly contractive self-mapping on $C$ with $\alpha \in[0,1)$. Denote by $C$ the collection of all weakly contractive mapping $\Phi$ on $C$. Let $0<\mu<2 \eta / k^{2}$ and $0<\gamma<\mu\left(\eta-\mu k^{2} / 2\right)=\tau$. Let the mapping $W_{n}$ be defined by (2.14). If $\left\{x_{n}\right\}$ is the sequence generated by $x_{1} \in C$ and

$$
\begin{gathered}
w_{n}=W_{n} x_{n}, \\
x_{n+1}=\alpha_{n} \gamma \Phi\left(x_{n}\right)+\left(I-\alpha_{n} \mu B\right) w_{n}, \quad \forall n \in \mathbb{N},
\end{gathered}
$$

where $\left\{\alpha_{n}\right\}$ is a sequence in $(0,1)$ which satisfies the following conditions $(C 1)-(C 3)$, then the sequence $\left\{x_{n}\right\}$ converges strongly to $x^{*}=P_{\Theta}(I-\mu B+\gamma \Phi)\left(x^{*}\right)$, which is the unique solution of the variational inequality

$$
\left\langle(\mu B-\gamma \Phi) x^{*}, x^{*}-x\right\rangle \leq 0, \quad \forall x \in \Theta
$$


which is the optimality condition for the minimization problem

$$
\min _{x \in F(T)} \frac{1}{2}\langle B x, x\rangle-h(x)
$$

where $h$ is a potential function for $\gamma \Phi$ (i.e., $h^{\prime}(x)=\gamma \Phi(x)$, for $x \in H$ ).

Proof. Taking $F_{j} \equiv 0$, for each $j=1,2, \ldots, M$ in Theorem 3.1, it is easy to obtain the desired conclusion.

Corollary 3.6. Let $C$ be a nonempty closed convex subset of a real Hilbert space $H$ such that $C \pm C \subset$ $C$, and let $S: C \rightarrow C$ be a nonexpansive mapping with $\Theta:=F(S) \neq \emptyset$. Let $B$ be a $k$-Lipschitzian and $\eta$-strongly monotone mapping on $C$ with $k>0, \eta>0$. Let $\Phi$ be a $\pi$-weakly contractive self-mapping on $C$ with $\alpha \in[0,1)$. Denote by $C$ the collection of all weakly contractive $\Phi$ on $C$. Let $0<\mu<2 \eta / k^{2}$ and $0<\gamma<\mu\left(\eta-\mu k^{2} / 2\right)=\tau$. If $\left\{x_{n}\right\}$ is the sequence generated by $x_{1} \in C$ and

$$
x_{n+1}=\alpha_{n} \gamma \Phi\left(x_{n}\right)+\left(I-\alpha_{n} \mu B\right) S x_{n}, \quad \forall n \in \mathbb{N},
$$

where $\left\{\alpha_{n}\right\}$ is a sequence in $(0,1)$ which satisfies the following conditions $(C 1)-(C 3)$, then the sequence $\left\{x_{n}\right\}$ converges strongly to $x^{*}=P_{\Theta}(I-\mu B+\gamma \Phi)\left(x^{*}\right)$, which is the unique solution of the variational inequality

$$
\left\langle(\mu B-\gamma \Phi) x^{*}, x^{*}-x\right\rangle \leq 0, \quad \forall x \in \Theta
$$

which is the optimality condition for the minimization problem

$$
\min _{x \in F(T)} \frac{1}{2}\langle B x, x\rangle-h(x)
$$

where $h$ is a potential function for $\gamma \Phi$ (i.e., $h^{\prime}(x)=\gamma \Phi(x)$, for $x \in H$ ).

Proof. Taking $F_{j} \equiv 0$, for each $j=1,2, \ldots, M$ and replacing $W_{n}$ by nonexpansive mapping $S$ in Theorem 3.1, it is easy to obtain the desired conclusion.

\section{An Example and Numerical Result}

In this section, we give a real simple numerical example of Theorem 3.1 as follows. 
Example 4.1. For simplicity, let $H=\mathbb{R}, C=[0,1], S_{n}=I . \quad F_{k}(x, y)=0$, for all $x, y \in H, r_{j, n}=$ $1, j \in 1,2,3, \ldots, M, B=I, f(x)=x^{2} /(1+x), \alpha_{n}=1 / n$ for every $n \in \mathbb{N}$ and $\mu=1$. Then $\left\{x_{n}\right\}$ is the sequence generated by

$$
x_{n+1}=\frac{x_{n}^{2}}{2 n\left(1+x_{n}\right)}+\left(1-\frac{1}{2 n}\right) x_{n}
$$

and $z \rightarrow 0$ as $n \rightarrow \infty$, where 0 is the unique solution of the minimization problem

$$
\min _{x \in C} x^{2}-x+\ln |x+1|+K
$$

where $K$ is a constant.

Proof. We divide the proof into 4 steps.

Step 1. Using the idea in [55], we can show that

$$
J_{r_{j, n}}^{F_{j}} x=P_{C} x, \quad \forall x \in H, j \in\{1,2, \ldots, M\},
$$

where

$$
P_{C} x= \begin{cases}\frac{x}{|x|}, & x \in H-C \\ x, & x \in C .\end{cases}
$$

Since $F_{j}(x, y)=0$, for all $x, y \in C, j \in\{1,2, \ldots, M\}$, with the definition of $J_{r}(x)$, for all $x \in H$ in Lemma 2.13, we have

$$
J_{r}^{F}(x)=\left\{z \in C: F(z, y)+\frac{1}{r}\langle y-z, z-x\rangle \geq 0, \forall y \in C\right\}
$$

By the equivalent property of the nearest projection $P_{C}$ from $H$ to $C$, we can conclude that if we take $x \in C, J_{r_{j, n}}^{F_{j}} x=P_{C} x=I x$. By (3) in Lemma 2.11, we have

$$
\bigcap_{j=1}^{M} \operatorname{EP}\left(F_{j}\right)=C .
$$

Step 2. We show that

$$
W_{n}=I .
$$


$S_{n}$ is nonexpansive mapping. By (2.14) we have

$$
\begin{aligned}
W_{1}=U_{1,1} & =\gamma_{1} S_{1} U_{1,2}+\left(1-\gamma_{1}\right) I, \\
W_{2}=U_{2,1} & =\gamma_{1} S_{1} U_{2,2}+\left(1-\gamma_{1}\right) I=\gamma_{1} S_{1}\left(\gamma_{2} S_{2} U_{2,3}+\left(1-\gamma_{2}\right) I\right)+\left(1-\gamma_{1}\right) I \\
& =\gamma_{1} \gamma_{2} S_{1} S_{2}+\gamma_{1}\left(1-\gamma_{2}\right) S_{1}+\left(1-\gamma_{1}\right) I, \\
W_{3}=U_{3,1} & =\gamma_{1} S_{1} U_{3,2}+\left(1-\gamma_{1}\right) I=\gamma_{1} S_{1}\left(\gamma_{2} S_{2} U_{3,3}+\left(1-\gamma_{2}\right) I\right)+\left(1-\gamma_{1}\right) I \\
& =\gamma_{1} \gamma_{2} S_{1} S_{2} U_{3,3}+\gamma_{1}\left(1-\gamma_{2}\right) S_{1}+\left(1-\gamma_{1}\right) I \\
& =\gamma_{1} \gamma_{2} S_{1} S_{2}\left(\gamma_{3} S_{3} U_{3,4}+\left(1-\gamma_{3}\right) I\right)+\gamma_{1}\left(1-\gamma_{2}\right) S_{1}+\left(1-\gamma_{1}\right) I \\
& =\gamma_{1} \gamma_{2} \gamma_{3} S_{1} S_{2} S_{3}+\gamma_{1} \gamma_{2}\left(1-\gamma_{3}\right) S_{1} S_{2}+\gamma_{1}\left(1-\gamma_{2}\right) S_{1}+\left(1-\gamma_{1}\right) I,
\end{aligned}
$$

and we compute (2.14) in the same way as above, so we obtain

$$
\begin{aligned}
W_{n}=U_{n, 1}= & \gamma_{1} \gamma_{2} \cdots \gamma_{n} S_{1} S_{2 n}+\gamma_{1} \gamma_{2} \cdots \gamma_{n-1}\left(1-\gamma_{n}\right) S_{1} S_{2 n-1} \\
& +\gamma_{1} \gamma_{2} \cdots \gamma_{n-2}\left(1-\gamma_{n-1}\right) S_{1} S_{2 n-2}+\cdots+\gamma_{1}\left(1-\gamma_{2}\right) S_{1}+\left(1-\gamma_{1}\right) I .
\end{aligned}
$$

Since $S_{n}=I, \gamma_{n}=\beta, n \in \mathbb{N}$, hence,

$$
W_{n}=\left[\beta^{n}+\beta^{n-1}(1-\beta)+\cdots+\beta(1-\beta)+(1-\beta)\right] I=I .
$$

Step 3. We prove

$$
x_{n+1}=\frac{x_{n}^{2}}{n\left(1+x_{n}\right)}+\left(1-\frac{1}{n}\right) x_{n}, \quad x_{n} \longrightarrow 0 \text { as } n \longrightarrow \infty,
$$

where 0 is the unique solution of the minimization problem

$$
\min _{x \in C} x^{2}-x+\ln |x+1|+K
$$

Since we let $B=I, \gamma$ be a real number, we choose $\gamma=1$. From (4.3), (4.4), and (4.7), we can obtain a special sequence $\left\{x_{n}\right\}$ of Theorem 3.1 as follows:

$$
x_{n+1}=\frac{x_{n}^{2}}{n\left(1+x_{n}\right)}+\left(1-\frac{1}{n}\right) x_{n}
$$

Since $S_{n}=I, n \in \mathbb{N}$, we have

$$
\bigcap_{n \in \mathbb{N}} F\left(S_{n}\right)=H
$$


Table 1: The sequence values on each different iteration steps.

\begin{tabular}{lccc}
\hline Iteration step $(n)$ & $x(1)=0.15$ & Iteration step $(n)$ & $x(1)=0.15$ \\
\hline 0 & 0.1500 & 7 & 0.0029 \\
1 & 0.0196 & 8 & 0.0025 \\
2 & 0.0100 & 9 & 0.0022 \\
3 & 0.0067 & $\vdots$ & $\vdots$ \\
4 & 0.0050 & 403 & 0.0001 \\
5 & 0.0040 & 404 & 0.0001 \\
6 & 0.0034 & 405 & 0.0000 \\
\hline
\end{tabular}

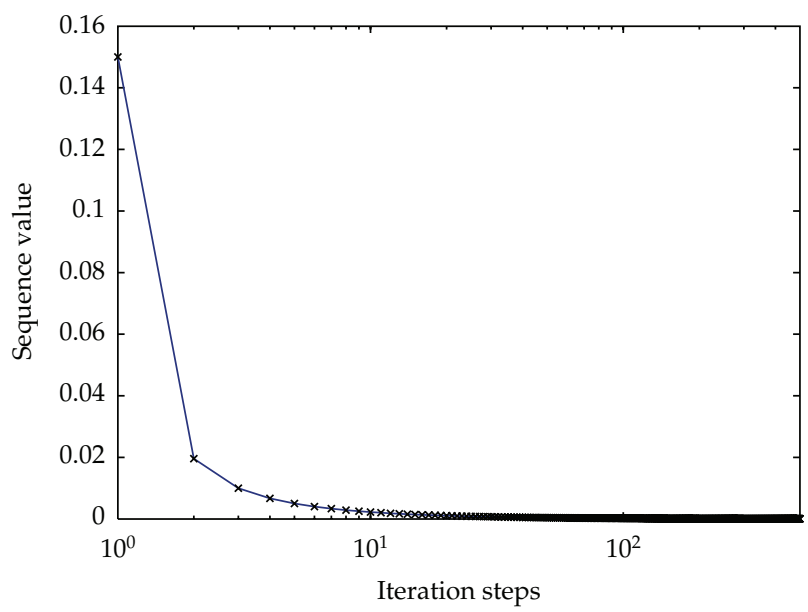

Figure 1: The initial value $x(1)=0.15$ and iteration steps $n=500$.

Combining with (4.6), we obtain

$$
\Theta:=\left(\bigcap_{j=1}^{M} \operatorname{EP}\left(F_{j}\right)\right) \cap\left(\bigcap_{i=1}^{\infty} F\left(S_{i}\right)\right)=C=[0,1] .
$$

It is obvious that $\left\{x_{n}\right\} \rightarrow 0$, and 0 is the unique solution of the minimization problem

$$
\min _{x \in C} x^{2}-x+\ln |x+1|+K
$$

where $K$ is a constant number.

\section{Numerical Result}

In this step, we give the numerical results (see Table 1) that support our main theorem as shown by the plotting graph using MATLAB 7.11.0. We choose the initial values as $x=0.15$ in Figure 1. From the example, we can see that $\left\{x_{n}\right\}$ converges to 0. 


\section{Acknowledgments}

This work was partially supported by the Higher Education Research Promotion and National Research University Project of Thailand, Office of the Higher Education Commission (NRU55-CSEC no. 55000613). Also, the first author would like to thank the Office of the Higher Education Commission, Thailand, for the financial support of the Ph.D. Program at KMUTT, and the second author was supported by Rajamangala University of Technology Lanna Research and Development Institute for the Ph.D. Program at KMUTT. Moreover, the third author was supported by the Higher Education Research Promotion and National Research University Project of Thailand, Office of the Higher Education Commission, for financial support during the preparation of this paper. Finally, the authors would like to thank the referees for their careful readings and valuable suggestions to improve the writing of this paper.

\section{References}

[1] Y. Censor, W. Chen, P. L. Combettes, R. Davidi, and G. T. Herman, "On the effectiveness of projection methods for convex feasibility problems with linear inequality constraints," Computational Optimization and Applications, vol. 51, no. 3, pp. 1065-1088, 2012.

[2] Y. Censor, A. Gibali, S. Rich, and S. Sabach, "Common solutions to variational inequalities," Set-Valued and Variational Analysis, vol. 20, no. 2, pp. 229-247, 2012.

[3] A. Auslender, Optimization-Méthodes Numériques, Masson, France, Paris, 1976.

[4] Y. Censor and S. Reich, "Iterations of paracontractions and firmly nonexpansive operators with applications to feasibility and optimization," Optimization, vol. 37, no. 4, pp. 323-339, 1996.

[5] Y. Censor, "Parallel application of block-iterative methods in medical imaging and radiation therapy," Mathematical Programming, vol. 42, no. 2, pp. 307-325, 1988.

[6] P. L. Combettes, "Hard-constrained inconsistent signal feasibility problems," IEEE Transactions on Signal Processing, vol. 47, no. 9, pp. 2460-2468, 1999.

[7] E. Blum and W. Oettli, "From optimization and variational inequalities to equilibrium problems," The Mathematics Student, vol. 63, no. 1-4, pp. 123-145, 1994.

[8] P. L. Combettes, "The convex feasibility problem in image recovery," in Advances in Imaging and Electron Physics, P. Hawkes, Ed., vol. 95, pp. 155-270, Academic Press, New York, NY, USA, 1996.

[9] P. L. Combettes and S. A. Hirstoaga, "Equilibrium programming in Hilbert spaces," Journal of Nonlinear and Convex Analysis, vol. 6, no. 1, pp. 117-136, 2005.

[10] O. Chadli, S. Schaible, and J. C. Yao, "Regularized equilibrium problems with application to noncoercive hemivariational inequalities," Journal of Optimization Theory and Applications, vol. 121, no. 3, pp. 571-596, 2004.

[11] O. Chadli, N. C. Wong, and J. C. Yao, "Equilibrium problems with applications to eigenvalue problems," Journal of Optimization Theory and Applications, vol. 117, no. 2, pp. 245-266, 2003.

[12] I. V. Konnov, S. Schaible, and J. C. Yao, "Combined relaxation method for mixed equilibrium problems," Journal of Optimization Theory and Applications, vol. 126, no. 2, pp. 309-322, 2005.

[13] A. Moudafi and M. Théra, "Proximal and dynamical approaches to equilibrium problems," in Ill-Posed Variational Problems and Regularization Techniques (Trier, 1998), vol. 477 of Lecture Notes in Econcmics and Mathematical Systems, pp. 187-201, Springer, Berlin, Germany, 1999.

[14] L. C. Zeng, S. Y. Wu, and J. C. Yao, "Generalized KKM theorem with applications to generalized minimax inequalities and generalized equilibrium problems," Taiwanese Journal of Mathematics, vol. 10, no. 6, pp. 1497-1514, 2006.

[15] F. Cianciaruso, G. Marino, L. Muglia, and Y. Yao, "A hybrid projection algorithm for finding solutions of mixed equilibrium problem and variational inequality problem," Fixed Point Theory and Applications, vol. 2010, Article ID 383740, 19 pages, 2010.

[16] P. Cholamjiak and S. Suantai, "A new hybrid algorithm for variational inclusions, generalized equilibrium problems, and a finite family of quasi-nonexpansive mappings," Fixed Point Theory and Applications, vol. 2009, Article ID 350979, 20 pages, 2009. 
[17] C. Jaiboon and P. Kumam, "Strong convergence for generalized equilibrium problems, fixed point problems and relaxed cocoercive variational inequalities," Journal of Inequalities and Applications, vol. 2010, Article ID 728028, 43 pages, 2010.

[18] C. Jaiboon, W. Chantarangsi, and P. Kumam, "A convergence theorem based on a hybrid relaxed extragradient method for generalized equilibrium problems and fixed point problems of a finite family of nonexpansive mappings," Nonlinear Analysis: Hybrid Systems, vol. 4, no. 1, pp. 199-215, 2010.

[19] P. Kumam and C. Jaiboon, "A new hybrid iterative method for mixed equilibrium problems and variational inequality problem for relaxed cocoercive mappings with application to optimization problems," Nonlinear Analysis: Hybrid Systems, vol. 3, no. 4, pp. 510-530, 2009.

[20] Y. Shehu, "Fixed point solutions of variational inequality and generalized equilibrium problems with applications," Annali dell'Universitá di Ferrara, Sezione 7, vol. 56, no. 2, pp. 345-368, 2010.

[21] Y. Yao, Y. C. Liou, and J. C. Yao, "A new hybrid iterative algorithm for fixed-point problems, variational inequality problems, and mixed equilibrium problems," Fixed Point Theory and Applications, vol. 2008, Article ID 417089, 15 pages, 2008.

[22] Y. Yao, Y. C. Liou, and Y. J. Wu, "An extragradient method for mixed equilibrium problems and fixed point problems," Fixed Point Theory and Applications, vol. 2009, Article ID 632819, 15 pages, 2009.

[23] J. C. Yao and O. Chadli, "Pseudomonotone complementarity problems and variational inequalities," in Handbook of Generalized Convexity and Generalized Monotonicity, vol. 76, pp. 501-558, Springer, Amsterdam, The Netherlands, 2005.

[24] L. C. Zeng, S. Schaible, and J. C. Yao, "Iterative algorithm for generalized set-valued strongly nonlinear mixed variational-like inequalities," Journal of Optimization Theory and Applications, vol. 124, no. 3, pp. 725-738, 2005.

[25] Y. Song, "Equivalent theorems of the convergence between proximal type algorithms," Nonlinear Analysis, vol. 71, no. 1-2, pp. 293-300, 2009.

[26] J. B. Diaz and F. T. Metcalf, "On the structure of the set of subsequential limit points of successive approximations," Bulletin of the American Mathematical Society, vol. 73, pp. 516-519, 1967.

[27] A. Moudafi, "Viscosity approximation methods for fixed-points problems," Journal of Mathematical Analysis and Applications, vol. 241, no. 1, pp. 46-55, 2000.

[28] H.-K. Xu, "Viscosity approximation methods for nonexpansive mappings," Journal of Mathematical Analysis and Applications, vol. 298, no. 1, pp. 279-291, 2004.

[29] G. Marino and H.-K. Xu, "A general iterative method for nonexpansive mappings in Hilbert spaces," Journal of Mathematical Analysis and Applications, vol. 318, no. 1, pp. 43-52, 2006.

[30] T. Suzuki, "Moudafi's viscosity approximations with Meir-Keeler contractions," Journal of Mathematical Analysis and Applications, vol. 325, no. 1, pp. 342-352, 2007.

[31] I. Yamada, "The hybrid steepest descent method for the variational inequality problem over the intersection of fixed point sets of nonexpansive mappings," in Inherently Parallel Algorithms in Feasibility and Optimization and Their Applications, D. Butnariu, Y. Censor, and S. Reich, Eds., vol. 8, pp. 473-504, Elservier, New York, NY, USA, 2001.

[32] M. Tian, "A general iterative algorithm for nonexpansive mappings in Hilbert spaces," Nonlinear Analysis, vol. 73, no. 3, pp. 689-694, 2010.

[33] S. Saeidi, "Modified hybrid steepest-descent methods for variational inequalities and fixed points," Mathematical and Computer Modelling, vol. 52, no. 1-2, pp. 134-142, 2010.

[34] S. S. Zhang, H. W. J. Lee, C. K. Chan, and J. A. Liu, "Viscosity approximation with weak contractions for fixed point problem, equilibrium problem, and variational inequality problem," Applied Mathematics and Mechanics (English Edition), vol. 31, no. 10, pp. 1273-1282, 2010.

[35] Y. I. Alber and S. Guerre-Delabriere, "Principle of weakly contractive maps in Hilbert spaces," in New Results in Operator Theory and Its Applications, I. Gohberg and Y. Lyubich, Eds., vol. 98 of Operator Theory: Advances and Applications, pp. 7-22, Birkhäuser, Basel, Switzerland, 1997.

[36] F. E. Browder, "Nonlinear operators and nonlinear equations of evolution in Banach spaces," Proceedings of Symposia in Pure Mathematics, vol. 18, pp. 78-81, 1976.

[37] G. L. Acedo and H.-K. Xu, "Iterative methods for strict pseudo-contractions in Hilbert spaces," Nonlinear Analysis, vol. 67, no. 7, pp. 2258-2271, 2007.

[38] Z. Opial, "Weak convergence of the sequence of successive approximations for nonexpansive mappings," Bulletin of the American Mathematical Society, vol. 73, pp. 591-597, 1967.

[39] W. Takahashi, Nonlinear Functional Analysis, Yokohama Publishers, Yokohama, Japan, 2000. 
[40] V. Colao, G. L. Acedo, and G. Marino, "An implicit method for finding common solutions of variational inequalities and systems of equilibrium problems and fixed points of infinite family of nonexpansive mappings," Nonlinear Analysis, vol. 71, no. 7-8, pp. 2708-2715, 2009.

[41] H. H. Bauschke, "The approximation of fixed points of compositions of nonexpansive mappings in Hilbert space," Journal of Mathematical Analysis and Applications, vol. 202, no. 1, pp. 150-159, 1996.

[42] C. E. Chidume and N. Shahzad, "Strong convergence of an implicit iteration process for a finite family of nonexpansive mappings," Nonlinear Analysis, vol. 62, no. 6, pp. 1149-1156, 2005.

[43] L. C. Ceng and J. C. Yao, "Hybrid viscosity approximation schemes for equilibrium problems and fixed point problems of infinitely many nonexpansive mappings," Applied Mathematics and Computation, vol. 198, no. 2, pp. 729-741, 2008.

[44] F. Deutsch and I. Yamada, "Minimizing certain convex functions over the intersection of the fixed point sets of nonexpansive mappings," Numerical Functional Analysis and Optimization, vol. 19, no. 1-2, pp. 33-56, 1998.

[45] X. Qin, M. Shang, and H. Zhou, "Strong convergence of a general iterative method for variational inequality problems and fixed point problems in Hilbert spaces," Applied Mathematics and Computation, vol. 200, no. 1, pp. 242-253, 2008.

[46] Y. Su and X. Qin, "General iteration algorithm and convergence rate optimal model for common fixed points of nonexpansive mappings," Applied Mathematics and Computation, vol. 186, no. 1, pp. 271-278, 2007.

[47] M. Shang, Y. Su, and X. Qin, "Strong convergencetheorems for a finite family of nonexpansive mappings," Fixed Point Theory and Applications, vol. 2007, Article ID 76971, 9 pages, 2007.

[48] K. Shimoji and W. Takahashi, "Strong convergence to common fixed points of infinite nonexpansive mappings and applications," Taiwanese Journal of Mathematics, vol. 5, no. 2, pp. 387-404, 2001.

[49] H.-K. Xu, "An iterative approach to quadratic optimization," Journal of Optimization Theory and Applications, vol. 116, no. 3, pp. 659-678, 2003.

[50] H.-K. Xu and R. G. Ori, "An implicit iteration process for nonexpansive mappings," Numerical Functional Analysis and Optimization, vol. 22, no. 5-6, pp. 767-773, 2001.

[51] Y. Yao, "A general iterative method for a finite family of nonexpansive mappings," Nonlinear Analysis, vol. 66, no. 12, pp. 2676-2687, 2007.

[52] Y. Yao, Y. C. Liou, and J. C. Yao, "Convergence theorem for equilibrium problems and fixed point problems of infinite family of nonexpansive mappings," Fixed Point Theory and Applications, vol. 2007, Article ID 64363, 12 pages, 2007.

[53] T.-H. Kim and H.-K. Xu, "Strong convergence of modified Mann iterations," Nonlinear Analysis, vol. 61, no. 1-2, pp. 51-60, 2005.

[54] V. Colao, G. Marino, and H.-K. Xu, "An iterative method for finding common solutions of equilibrium and fixed point problems," Journal of Mathematical Analysis and Applications, vol. 344, no. 1, pp. 340$352,2008$.

[55] H. He, S. Liu, and Y. J. Cho, "An explicit method for systems of equilibrium problems and fixed points of infinite family of nonexpansive mappings," Journal of Computational and Applied Mathematics, vol. 235, no. 14, pp. 4128-4139, 2011. 


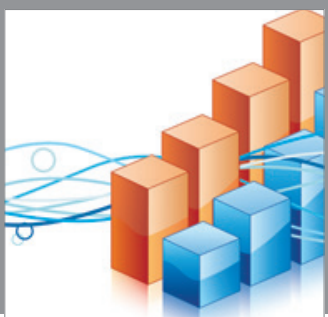

Advances in

Operations Research

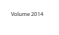

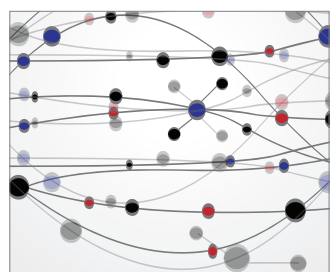

\section{The Scientific} World Journal
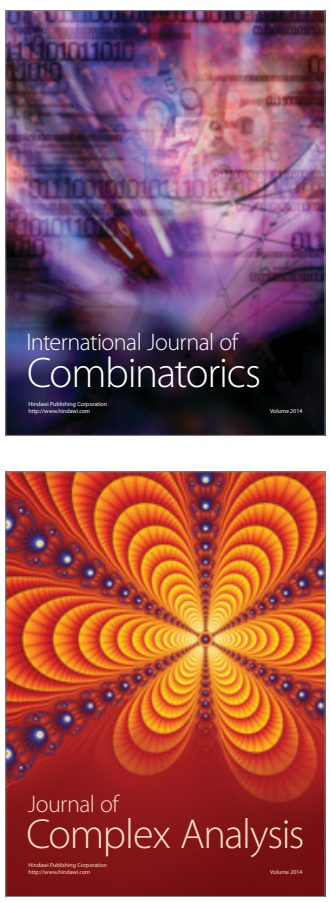

International Journal of

Mathematics and

Mathematical

Sciences
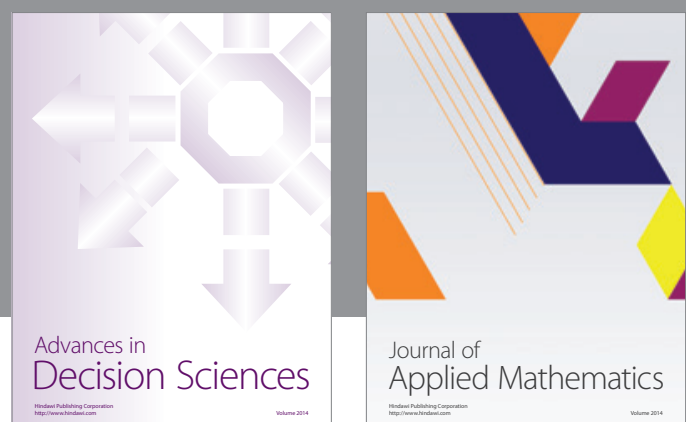

Journal of

Applied Mathematics
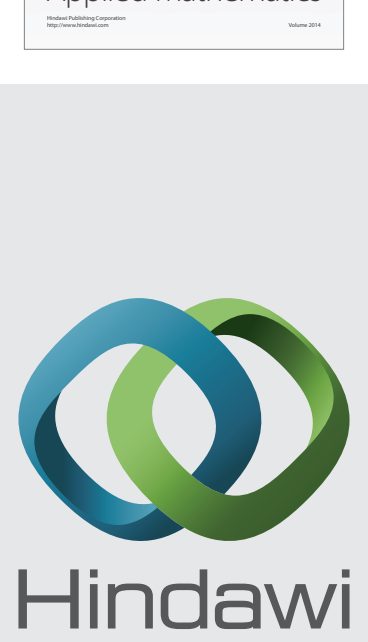

Submit your manuscripts at http://www.hindawi.com
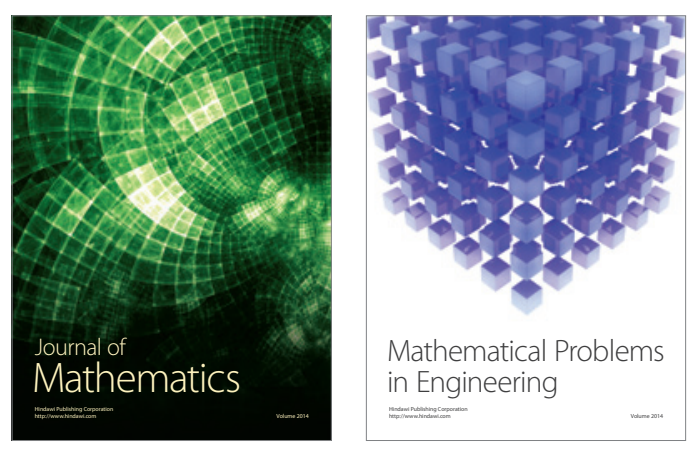

Mathematical Problems in Engineering
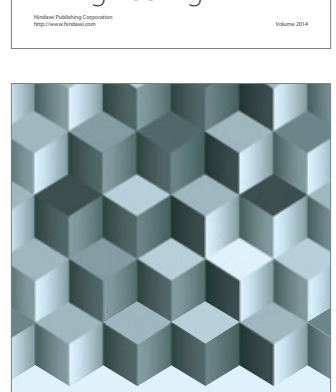

Journal of

Function Spaces
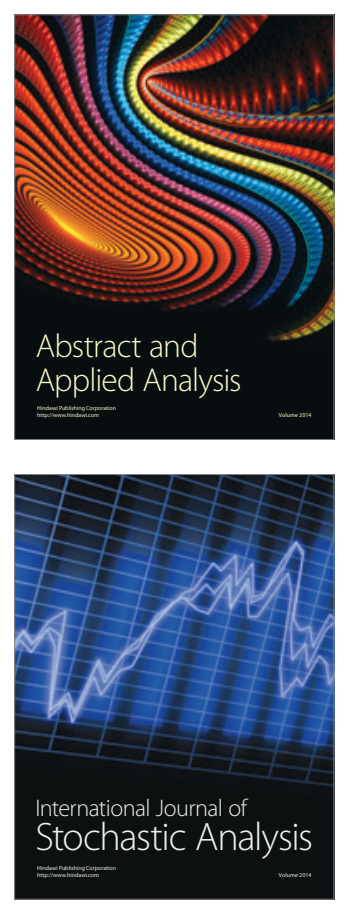

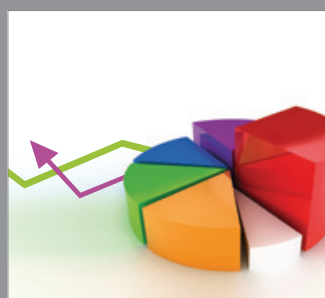

ournal of

Probability and Statistics

Promensencen
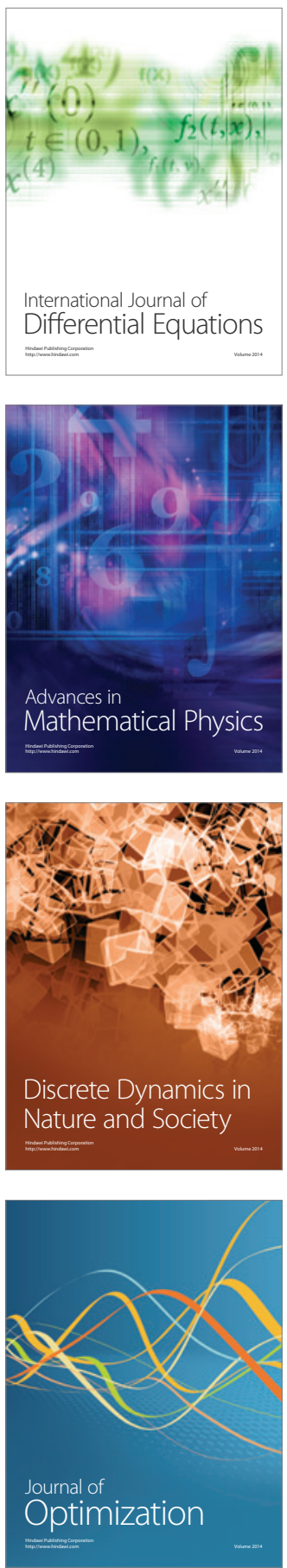